\title{
A Millimeter-wave Galactic Plane Survey with the BICEP Polarimeter
}

\section{Citation}

Bierman, E. M, J. Kovac et al. 2011. "A Millimeter-Wave Galactic Plane Survey with the BICEP Polarimeter." The Astrophysical Journal 741: 81.

\section{Published Version}

doi:10.1088/0004-637X/741/2/81

\section{Permanent link}

http://nrs.harvard.edu/urn-3:HUL.InstRepos:12712844

\section{Terms of Use}

This article was downloaded from Harvard University's DASH repository, and is made available under the terms and conditions applicable to Other Posted Material, as set forth at http:// nrs.harvard.edu/urn-3:HUL.InstRepos:dash.current.terms-of-use\#LAA

\section{Share Your Story}

The Harvard community has made this article openly available.

Please share how this access benefits you. Submit a story.

Accessibility 


\title{
A MILLIMETER-WAVE GALACTIC PLANE SURVEY WITH THE BICEP POLARIMETER
}

\author{
E. M. Bierman ${ }^{1}$, T. Matsumura ${ }^{2}$, C. D. Dowell ${ }^{2,3}$, B. G. Keating $^{1}$, P. Ade ${ }^{4}$, D. Barkats ${ }^{5}$, D. Barron ${ }^{1}$, J. O. Battle ${ }^{3}$, \\ J. J. Bock ${ }^{2,3}$, H. C. Chiang ${ }^{2,6}$, T. L. Culverhouse ${ }^{2}$, L. Duband ${ }^{7}$, E. F. Hivon ${ }^{8}$, W. L. Holzapfel ${ }^{9}$, V. V. Hristov ${ }^{2}$,

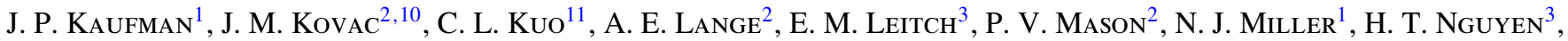 \\ C. Pryke ${ }^{12}$, S. Richter ${ }^{2}$, G. M. Rocha ${ }^{2}$, C. Sheehy ${ }^{12}$, Y. D. TAKahashi ${ }^{9}$, and K. W. Yoon ${ }^{13}$ \\ ${ }^{1}$ University of California, San Diego, USA; ebierman@ physics.ucsd.edu \\ ${ }^{2}$ California Institute of Technology, USA \\ ${ }^{3}$ Jet Propulsion Laboratory, USA \\ ${ }^{4}$ University of Wales, UK \\ 5 Joint ALMA Office-NRAO, Chile \\ ${ }^{6}$ Princeton University, USA \\ ${ }^{7}$ Commissariat à l'Énergie Atomique, France \\ ${ }^{8}$ Institut d'Astrophysique de Paris, France \\ ${ }^{9}$ University of California, Berkeley, USA \\ ${ }^{10}$ Harvard University, USA \\ ${ }^{11}$ Stanford University, CA, USA \\ ${ }^{12}$ University of Chicago, USA \\ ${ }^{13}$ National Institute of Standards and Technology, USA \\ Received 2011 February 23; accepted 2011 August 3; published 2011 October 20
}

\begin{abstract}
In order to study inflationary cosmology and the Milky Way Galaxy's composition and magnetic field structure, Stokes $I, Q$, and $U$ maps of the Galactic plane covering the Galactic longitude range $260^{\circ}<\ell<340^{\circ}$ in three atmospheric transmission windows centered on 100,150 , and $220 \mathrm{GHz}$ are presented. The maps sample an optical depth $1 \lesssim A_{V} \lesssim 30$, and are consistent with previous characterizations of the Galactic millimeter-wave frequency spectrum and the large-scale magnetic field structure permeating the interstellar medium. The polarization angles in all three bands are generally perpendicular to those measured by starlight polarimetry as expected and show changes in the structure of the Galactic magnetic field on the scale of $60^{\circ}$. The frequency spectrum of degree-scale Galactic emission is plotted between 23 and $220 \mathrm{GHz}$ (including WMAP data) and is fit to a two-component (synchrotron and dust) model showing that the higher frequency BICEP data are necessary to tightly constrain the amplitude and spectral index of Galactic dust. Polarized emission is detected over the entire region within two degrees of the Galactic plane, indicating the large-scale magnetic field is oriented parallel to the plane of the Galaxy. A trend of decreasing polarization fraction with increasing total intensity is observed, ruling out the simplest model of a constant Galactic magnetic field orientation along the line of sight in the Galactic plane. A generally increasing trend of polarization fraction with electromagnetic frequency is found, varying from $0.5 \%-1.5 \%$ at frequencies below $50 \mathrm{GHz}$ to $2.5 \%-3.5 \%$ above $90 \mathrm{GHz}$. The effort to extend the capabilities of BICEP by installing $220 \mathrm{GHz}$ band hardware is described along with analysis of the new band.
\end{abstract}

Key words: cosmology: observations - Galaxy: structure - instrumentation: polarimeters - radio continuum: ISM - submillimeter: diffuse background - submillimeter: ISM

Online-only material: color figures

\section{INTRODUCTION}

Emission from the Milky Way Galaxy at millimeter wavelengths is both a rich source of astrophysical information and a potential contaminant for cosmic microwave background (CMB) observations. The density of gas and dust in the interstellar medium (ISM) varies from very low $\left(<1\right.$ particle $\left.\mathrm{cm}^{-3}\right)$ in diffuse regions to very high $\left(>10^{6}\right.$ particle $\left.\mathrm{cm}^{-3}\right)$ in molecular clouds and complexes (collections of star-forming cloud cores at approximately the same distance and age). Measurements in the millimeter band have the potential to probe a wide range of ISM densities while near-IR bands (Martin \& Whittet 1990) have enough resolution to probe medium to high density ISM regions.

In general, large-scale diffuse emission from the Galaxy at frequencies below $90 \mathrm{GHz}$ is dominated by the synchrotron mechanism in ionized gas, and to a lesser extent free-free along with thermal emission from rotational and vibrational modes of dust (Rybicki \& Lightman 1979). Emission at frequencies above $90 \mathrm{GHz}$ is dominated by vibrational modes of Galactic dust (Whittet 1992). However, not all emission mechanisms give rise to polarized radiation. For example, free-free emission from electron-ion scattering can contribute to the measured intensity (although it is not the dominant ore at any millimeter-wave band) near $90 \mathrm{GHz}$; however, this emission is not polarized. The exact composition of the Galaxy's emission spectrum varies across the sky and can change when observing polarized intensity. Multi-wavelength observations in the infrared and millimeter can determine the properties of the continuum emission, spectral lines, and polarization.

Polarized radiation probes various aspects of the ISM and Galactic magnetic field. Dust grains aligned perpendicular to the Galactic plane by the Galactic magnetic field preferentially absorb and scatter starlight in one direction, causing more extinction of one polarization mode compared to the other (Davis \& Greenstein 1951; Hiltner 1951; Hildebrand 1988; Fosalba et al. 2002; Lazarian 2007). Complementary to this, dust grains aligned by the Galactic magnetic field emit radiation 
in the infrared and millimeter bands polarized orthogonally to the Galactic magnetic field (Lazarian 2007). The desire to characterize the ISM and the Galactic magnetic field provides the motivation for millimeter-wave continuum polarimetry of the Galaxy (Hildebrand et al. 1999; Chuss et al. 2003; Novak et al. 2003; Benoît et al. 2004; Matthews et al. 2009; Culverhouse et al. 2010; Dotson et al. 2010). The main goal of the Background Imager of Cosmic Extragalactic Polarization (BICEP; Keating et al. 2003a; Chiang et al. 2010) and its successors ${ }^{14}$ is to search for the unique CMB B-mode polarization pattern due to primordial gravitational waves, which has an amplitude determined by the energy scale of inflation (Seljak \& Zaldarriaga 1997; Kamionkowski et al. 1997). Polarized Galactic emission is an astronomical foreground (Bock et al. 2008) that may need to be confronted to make this measurement, and therefore, the properties of the emission also motivate the investigation (Jones 2003; Ponthieu et al. 2005; Tucci et al. 2005; Eriksen et al. 2006; Hansen et al. 2006; Amblard et al. 2007; Larson et al. 2007; Eriksen et al. 2008; Leach et al. 2008; Miville-Deschênes et al. 2008; Dodelson et al. 2009; Dunkley et al. 2009). Only a few experiments have explored large-scale Galactic polarization properties at frequencies between $90 \mathrm{GHz}$ and $350 \mathrm{GHz}$, such as Archeops (353 GHz; Benoît et al. 2004), WMAP (94 GHz; Kogut et al. 2007), and QUaD (100 and $150 \mathrm{GHz}$; Culverhouse et al. 2010). To further study emission from sources other than the CMB, BICEP was upgraded from a two-band experiment (100 and $150 \mathrm{GHz}$ ) to a three-band polarimeter with the addition of $220 \mathrm{GHz}$ capability for the second and third seasons. This paper discusses unique aspects of the BICEP Galactic observations, including the Galactic maps with the additional $220 \mathrm{GHz}$ channels (Section 3.1), explains the data analysis methodology (Sections 2.2-2.5), and discusses the analysis of the polarized Galactic signal (Sections 3.6 and 3.7).

\section{BICEP INSTRUMENT AND MAPMAKING}

\subsection{Brief Instrument Description}

For a complete description of the BICEP telescope, see Yoon et al. (2006) and Takahashi et al. (2010). BICEP is an on-axis refracting telescope with a $250 \mathrm{~mm}$ aperture and can scan in azimuth and elevation as well as rotate around the optical axis (boresight) of the telescope, which is less than 0.01 away from the center feed. BICEP's small aperture allows the entire optical system to be cooled to cryogenic temperatures in a vacuum cryostat, sealed with a millimeter-wave transparent foam window. While located at the South Pole, the telescope mount is enclosed at room temperature within the observatory, protected by a fabric bellows structure. To control the response of the beam sidelobes and minimize ground contamination, the telescope has an inner co-moving absorptive forebaffle and a fixed reflective outer ground screen, similar to that used in POLAR (Polarization Observations of Large Angular Regions; Keating et al. 2003b). The readout electronics are sealed in an RF-tight cage and consist of detector AC biasing circuits, analog preamplifiers, lock-in amplifier cards, and cold JFETs in the cryostat.

The focal plane is comprised of 49 pairs of polarization sensitive bolometers (PSBs; Jones et al. 2003), two orthogonal detectors per feed, whose responses are summed or differenced to measure total intensity and polarization, respectively. For the first observing season in 2006, the focal plane had twenty-five

\footnotetext{
14 For a list of future space, balloon, and ground CMB experiments, see http://cmbpol.uchicago.edu/workshops/technology2008/depot/ meyer-stephan.pdf.
}

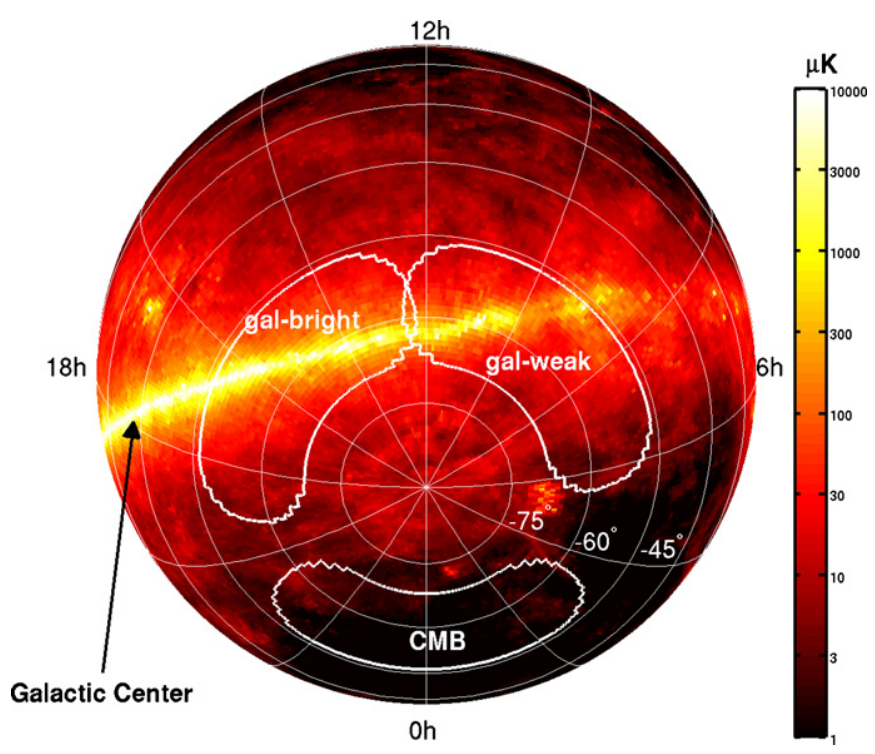

Figure 1. $150 \mathrm{GHz}$ FDS Model 8 dust emission prediction (Finkbeiner et al. 1999), shown in equatorial coordinates. BICEP's primary CMB observing field is called "the southern Galactic hole," a region of low dust emission used for optimal B-mode detection. The two Galactic fields are used for studying astronomical foregrounds and Galactic physics. The Gal-weak region spans the Galactic plane from Galactic longitude $260^{\circ}<\ell<300^{\circ}$, while the Gal-bright region spans the plane from $300^{\circ}<\ell<340^{\circ}$.

(A color version of this figure is available in the online journal.)

feeds tuned for the $100 \mathrm{GHz}$ atmospheric transmission window and twenty-four tuned for the $150 \mathrm{GHz}$ atmospheric window. For the second and third observing seasons, the focal plane consisted of twenty-five $100 \mathrm{GHz}$ feeds, twenty-two $150 \mathrm{GHz}$ feeds, and two new feeds tuned for the $220 \mathrm{GHz}$ atmospheric transmission window (discussed in Appendix A). Three corrugated feedhorn sections, cooled to $\leqslant 4 \mathrm{~K}$, couple radiation from the two highdensity polyethylene lenses to each PSB while also providing a sharp low-frequency cutoff for the band. The high-frequency cutoff is defined by a set of metal mesh filters (Ade et al. 2006) attached to the feedhorn stack. The filters and PSBs are cooled to $250 \mathrm{mK}$ by an ${ }^{4} \mathrm{He}-{ }^{3} \mathrm{He}-{ }^{3} \mathrm{He}$-sorption refrigerator system (Duband et al. 1990). Teflon filters block out-of-band infrared radiation to minimize optical loading.

\subsection{Scan Strategy}

Figure 1 shows the three main BICEP observing regions overlaid on the Galactic dust model of (Finkbeiner et al. 1999, hereafter FDS) evaluated at $150 \mathrm{GHz}$. Approximately $10,000 \mathrm{hr}$ were spent observing a region predicted to have minimal astronomical foreground contamination in an attempt to detect the B-mode signature of inflationary gravitational waves ("CMB" region), $945 \mathrm{hr}$ were dedicated to observing the Galactic plane in a region near the center of the Galaxy ("Gal-bright" region, 300 $<\ell<340^{\circ}$ ) and $1484 \mathrm{hr}$ were spent observing the Galactic plane in a region farther from the Galactic center ("Gal-weak" region, $260^{\circ}<\ell<300^{\circ}$ ).

BICEP observes all regions in a similar manner. The smallest observing unit is a "half-scan", a unidirectional azimuthal telescope movement at constant elevation that lasts $27 \mathrm{~s}(10 \mathrm{~Hz}$ sample rate). To avoid potential thermal disturbances, $3.5 \mathrm{~s}$ are cut at the beginning and end of each half-scan. The choice of scan speed is bounded at low frequency by the atmosphere and detector stability, and bounded at high frequency by the bolometer time constant. Within those constraints, the telescope scan 
speed is chosen to be $2.8 \mathrm{~s}^{-1}$ in azimuth to minimize microphonics and thermal drifts. A "scan-set" lasts approximately one hour and consists of 50 half-scans each in the positive and negative azimuthal directions at a given elevation.

A calibration period at the beginning and end of each scan-set consists of a small, one-degree elevation movement, called an "el-nod," which serves as the primary relative gain calibration within a feed and across the focal plane. Atmospheric loading is proportional to the line-of-sight air mass, which is well modeled as $\csc \left(\theta_{\mathrm{el}}\right)$ plus an offset in the readout electronics, where $\theta_{\mathrm{el}}$ is the elevation angle. El-nods produce a similar detector response (approximately $100 \mathrm{mK}$ peak to peak) across all detectors for an elevation change of one degree. To normalize the response over time, the average response during the el-nod for all the detectors in each band is calculated and divided out.

As opposed to tracking the celestial observing center, BICEP centers each scan-set about a fixed azimuth angle causing sky sources to move relative to the scan center, while stationary ground and scan thermal/optical contamination remains fixed. This has the added benefit of grouping both the scan and ground contaminations into one contamination ("scan-fixed contamination"). Chiang et al. (2010) take this process a step further and remove a template of the scan-fixed contamination from each scan-set; however, this additional step was not necessary in this paper because of the much larger polarization signal relative to the noise.

After each scan-set, the telescope is stepped in elevation by 0.25 and moved in azimuth to locate the next scan-set about the center of the observing region. Each set of scan-sets (called a "phase") consists of seven (lasting six hours) or ten (lasting nine hours) steps at one of four orientations about the boresight $\left(0^{\circ}\right.$, $180^{\circ}, 135^{\circ}, 315^{\circ}$ ) centered in the elevation range $55^{\circ}$ to $60^{\circ}$. Observations of the Gal-weak field were carried out mostly in six-hour phases in Austral winter. Observations of the Gal-bright field consisted primarily of nine-hour phases during Austral summer 2008, although there were a few other six-hour phases executed at various times during the three years of observing.

Timestream statistics (such as the variance, skew, and kurtosis of a half-scan) and el-nod calibration values are used to determine nominal observing conditions. These statistics allowed the data to be cut on various timescales such as per-phase, per-scanset, and per-half-scan bases. Gal-bright observations use 763 out of 945 total possible hours and Gal-weak observations use 1463 out of 1484 total possible hours based on el-nod cuts, while scan statistics cut approximately $5 \%$ of the remaining data.

\subsection{Spectroscopic Characterization}

Instrumental properties of the telescope can cause noticeable effects in the observed Galaxy maps unless they are properly taken into account. One of the most pernicious of these properties is the spectral response mismatch, which can affect the resulting maps differently depending upon the observed source's spectrum. The key instrumental properties of BICEP are summarized in Table 1 as described in this section, Appendix B, and Takahashi et al. (2010).

For all PSBs used in BICEP, at all three frequency bands, detected radiation from the sky produces a bolometer signal,

$$
\begin{aligned}
d(t)= & K(t) \otimes\left\{n(t)+g(t) \int d v A_{e}(v) F(v)\right. \\
& \times \int d \Omega P(\Omega)(I+\gamma(Q \cos (2 \psi)+U \sin (2 \psi))\}
\end{aligned}
$$

Table 1

\begin{tabular}{|c|c|c|c|}
\hline Instrument Property (Band Average) & 100 & 150 & 220 \\
\hline Number of Feeds $(2006,2007-2008)$ & 25,25 & 24,22 & 0,2 \\
\hline Polarization Orientation Uncertainty & $<0.7$ & $<0.7$ & $<0.7$ \\
\hline Pair-relative Polarization Orientation Uncertainty & 0.1 & 0.1 & 0.1 \\
\hline Polarization Efficiency, $\gamma$ & 0.92 & 0.93 & 0.85 \\
\hline ptical Efficiency & $20.8 \%$ & $19.8 \%$ & $15.8 \%$ \\
\hline aussian Beam Width (FWHM) & 0.93 & 0.60 & 0.42 \\
\hline ifferential Pointing/Beam Size & $1.0 \%$ & $1.8 \%$ & $2.6 \%$ \\
\hline host Beam Power & $0.41 \%$ & $0.50 \%$ & $1.3 \%$ \\
\hline Ghost Beam Power, Pair-Difference & $0.02 \%$ & $0.04 \%$ & $0.04 \%$ \\
\hline Spectral Band Centers, flat source $(\mathrm{GHz})^{\mathrm{a}}$ & 95.5 & 149.8 & 208.2 \\
\hline Spectral Band Centers, Galaxy (GHz) & 96.3 & 152.4 & 212.2 \\
\hline Spectral Gain Mismatch ${ }^{\mathrm{b}}$ & $0.17 \%$ & $0.19 \%$ & $0.72 \%$ \\
\hline Relative Gain Uncertainty ${ }^{\mathrm{b}}$ & $0.8 \%$ & $1.3 \%$ & $<10 \%$ \\
\hline 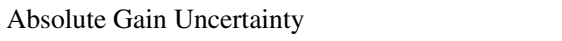 & $2 \%$ & $2 \%$ & $15 \%$ \\
\hline uivalent Temperature $(\mu \mathrm{K} \sqrt{s})$ & 530 & 450 & 1040 \\
\hline$\left(\mathrm{MJy} \mathrm{sr}^{-1} \sqrt{s}\right)$ & 0.12 & 0.18 & 0.50 \\
\hline Noise Equivalent Q-Polarizat & 410 & 340 & 880 \\
\hline$\left(\mathrm{MJy} \mathrm{sr}^{-1} \sqrt{s}\right)$ & 0.090 & 0.14 & 0.42 \\
\hline
\end{tabular}

Telescope Characteristics

Notes. Characteristics of BICEP from its three observing seasons. While not an exhaustive list of all possible categories and errors, the listed parameters show the properties of the polarimeter relevant for this paper. Section 2.3 discusses the spectroscopic characterizations in more detail, while a brief discussion of the other BICEP characterizations are in Appendix B and Section 3.3.4 with further discussion in Takahashi et al. (2010). Characteristics for 100 and $150 \mathrm{GHz}$ are consistent with Takahashi et al. (2010) except for spectral characterizations.

a These values are slightly different for 100 and $150 \mathrm{GHz}$ from Takahashi et al. (2010) due to measurement uncertainties.

b These values are computed before correcting for spectral gain mismatch from FTS measurements.

${ }^{c}$ These are the single Stokes parameter noise values per feed used throughout this paper to calculate the white-noise levels of the maps.

where $I, Q$, and $U$ are the total intensity and two linear polarization Stokes parameters on the sky, respectively. BICEP is incapable of measuring the fourth Stokes parameter $V$, which accounts for circular polarization. However, for the CMB and Galaxy emission, $V$ is expected to be negligible compared with the two linear Stokes parameters. The effective antenna area, $A_{e}$, is assumed to be proportional to $v^{-2}$. To recover the underlying sky signal from the detector voltage timestreams $d(t)$, each of the following parameters is calibrated as described in Takahashi et al. (2010): $\psi$, the detector polarization angle; $\epsilon$, the cross-polarization response; $\gamma=\frac{1-\epsilon}{1+\epsilon}$, the resulting polarization efficiency; $P(\Omega)$, the antenna response as a function of angular position $\Omega ; F(v)$, the end-to-end detector spectral response including filters, feedhorns, lenses, etc.; $g(t)$, the detector responsivity; $n(t)$, the noise; $K(t)$, the time-domain bolometer transfer function and filtering due to the electronics that is convolved (" $\otimes$ ") with the detector signal.

Takahashi et al. (2010) explored the leakage of total intensity to polarization for each feed, estimated using individual PSB pair-sum and pair-difference maps from the CMB region. The relative gain uncertainty is similar to spectral gain mismatch but can include other effects such as thermal response mismatch. The maps were cross-correlated, showing the relative gain uncertainty was less than $0.8 \%$ and $1.3 \%$ for 100 and $150 \mathrm{GHz}$ feeds, respectively. It was found that the relative gain mismatch for the $220 \mathrm{GHz}$ pixels was $10 \%$ and a visual inspection of the maps showed the two $220 \mathrm{GHz}$ detectors gave inconsistent polarization results. The cause of this inconsistency was found to be mostly attributable to spectral gain mismatch. 




Figure 2. BICEP's three electromagnetic frequency spectra, $F(v)$, normalized to unity (black) with the band centers (shown by vertical purple dashed lines). Also plotted is the average spectral response for WMAP's $94 \mathrm{GHz}$ band normalized to 0.5 (gray) and the spectral radiance, $S(v)$, for a model of atmospheric emission at the South Pole ${ }^{16}$ (red), the spectrum (blue) of the CMB anisotropy (temperature derivative of the Planck function evaluated at 2.725 K; Fixsen \& Mather 2002) and a typical Galactic emission spectrum (green) in the BICEP analysis region, as seen through the atmosphere.

(A color version of this figure is available in the online journal.)

A careful campaign to characterize all of BICEP's feeds was undertaken in 2008 January at the South Pole using a highresolution $(250 \mathrm{MHz})$ polarized Fourier transform spectrometer (FTS). The optical alignment and power falling on each pair of bolometers in a feed was calibrated before a set of eight independent spectral measurements were taken. Figure 2 shows $F(v)$, the average spectral responses for each band with the FTS's source spectrum divided out, assuming the FTS's source filled the pixel's beam.

If not calibrated properly, mismatched spectral responses can cause spurious polarization in detector differences and gain errors among different feeds. For example, the WMAP satellite (Jarosik et al. 2007) notes that passband mismatch is a problem, solved by fitting for a spurious map component. The value of the mismatch is quoted as $1 \%$ on average with a maximum of $3.5 \%$ for the $23 \mathrm{GHz}$ band (Page et al. 2007), in agreement with preflight spectral measurements. BICEP does not have sufficient polarization angle coverage for each feed to fit out the spurious component; however, it is possible to use the measured spectral responses to mitigate this effect.

BICEP's spectral response mismatch leaks intensity into polarization and is caused by the combination of two effects. First, several feeds have mismatched spectra, most notably the two $220 \mathrm{GHz}$ feeds. Second, each PSB is calibrated relatively using the change in atmospheric loading with elevation; however, atmospheric emission has a different spectrum than Galactic emission or the CMB. The spectral mismatch leakage is calculated using the measured instrumental spectral response, a model of the atmospheric emission at the South Pole, ${ }^{15}$ and a model of the typical Galactic spectrum in the $220 \mathrm{GHz}$ observing region derived from fitting Equation (18) to BICEP and WMAP data as performed in Section 3.5.

The spectral gain mismatch $(\xi)$ is

$$
\xi=\frac{G_{A}-G_{B}}{G_{A}+G_{B}}
$$

\footnotetext{
15 Smithsonian Astrophysical Observatory, https://www.cfa.harvard.edu/ spaine/am/.
}

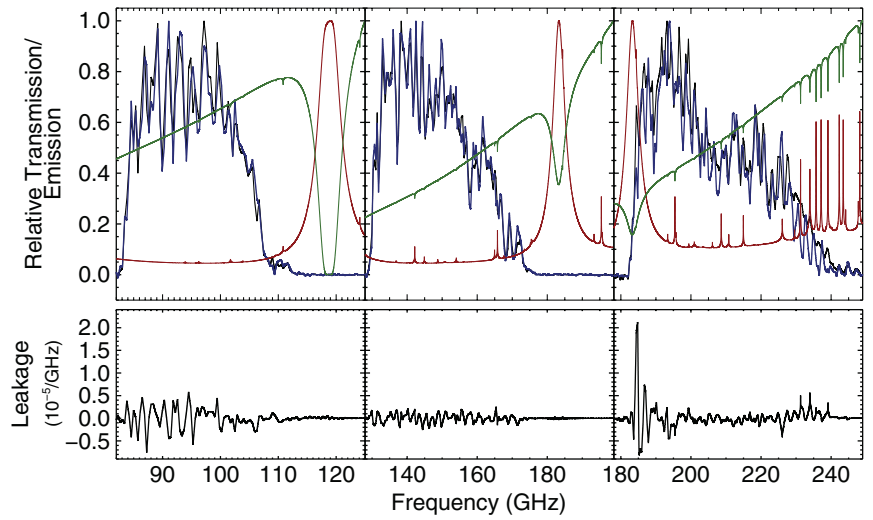

Figure 3. Spectral gain mismatch for one feed at each of BICEP's three bands. The top plots show the measured spectral responses for the two PSBs in a feed, "A" (black) and "B" (blue), along with a model of atmospheric transmission at the South Pole during median Austral winter conditions (red) and a typical Galactic source spectrum (green) in the $220 \mathrm{GHz}$ analysis region, as seen through the atmosphere. The bottom three plots show the leakage fraction per frequency, $d \xi / d \nu$, if calibrated off the atmospheric emission but observing the Galactic source spectrum as shown.

(A color version of this figure is available in the online journal.)

where the responsivities, $G_{A, B}$, represent the two PSBs in a feed and are given by

$$
\begin{gathered}
G_{A, B}=\frac{\Gamma_{\text {atmosphere }}}{\Gamma_{\text {Galaxy }}}, \\
\Gamma_{\text {source }}=\int F(v) S(v) v^{-2} d v,
\end{gathered}
$$

where $S(v)$ is the spectral radiance $\left(W / s r m^{2} \mathrm{~Hz}\right)$ of the source and $v^{-2}$ accounts for the throughput of the optics, which are assumed to be single-moded.

Shown in Figure 3 are examples of the spectral gain mismatch between two PSBs in a feed for each band. For the purpose of this paper, the spectral gain mismatch per frequency quantity, "leakage fraction," is defined by

$$
\frac{d \xi}{d \nu} \equiv \frac{\left(\frac{F_{A}(v)}{\Gamma_{A, \text { Galaxy }}}-\frac{F_{B}(v)}{\Gamma_{B, \text { Galaxy }}}\right) S_{\text {atmosphere }}(\nu) v^{-2}}{\left(G_{A}+G_{B}\right)}
$$

The integral of the leakage fraction, $d \xi / d \nu$, over the bounds shown gives the total spectral leakage measured for that feed, $\xi$. The leakage at $220 \mathrm{GHz}$ comes from the combination of a small difference in lower band edge and a large amount of power from the atmospheric water line at $185 \mathrm{GHz}$. The average magnitudes of the spectral gain mismatch are $0.17 \%, 0.19 \%, 0.72 \%$ for 100 , 150 , and $220 \mathrm{GHz}$, respectively, using a typical Galactic source spectrum and median precipitable water vapor conditions at the South Pole during Austral winter. Changing the observed source or atmospheric conditions can have an appreciable change in these numbers. For example, one of the $150 \mathrm{GHz}$ feeds has a $0.12 \%$ leakage during typical Austral summer conditions but can change in value by $0.5 \%$ depending on the atmospheric model. In this paper, typical values are used to correct the maps for this effect and simulations are carried out to probe the effects of the uncertainty in this calculated parameter.

The absolute gain calibration for BICEP maps at each frequency, co-added over all detectors, is determined by comparing total intensity angular cross power spectra from the BICEP 
$\mathrm{CMB}$ region to the angular power spectra found by WMAP in the same region (see Chiang et al. 2010 and Takahashi et al. 2010 for more details). Spectral gain mismatch between feeds could potentially introduce systematic effects into the analysis via feed calibration differences. Comparing the two $220 \mathrm{GHz}$ feed intensity maps, a difference of $30 \%$ is found. While this discrepancy was initially suspected to be due to spectral gain mismatch between different feeds, an investigation into the origin of this discrepancy did not find that this was the cause. While calibration per feed is important, this effect does not leak intensity power to polarization, and the quantities studied in this paper are mostly relative quantities insensitive to this systematic. Therefore, a calibration error per feed will not significantly affect the results of this paper.

While the three bands are called "100 GHz," "150 GHz," and "220 GHz," these are not the actual band centers. The band center for a given source is calculated as

$$
v_{0}=\frac{\int_{v_{L}}^{v_{H}} v F(v) S(v) v^{-2} d v}{\int_{v_{L}}^{v_{H}} F(v) S(v) v^{-2} d v},
$$

where $F(v)$ is the measured average spectral response for each band, $S(v)$ is the source emission spectrum, and $v^{-2}$ accounts for the throughput of the receiver. For a flat spectral source $S(v)=$ constant, this gives $95.5,149.8$, and $208.2 \mathrm{GHz}$ for 100 , 150 , and $220 \mathrm{GHz}$ bands, respectively. The dominant source of uncertainty is due to the optical setup and whether the FTS source is beam filling or not. This can change these values by $0.5,0.8$, and $1.0 \mathrm{GHz}$ for 100,150 , and $220 \mathrm{GHz}$, respectively. Changing the integration limits can also change these values by approximately $0.1 \mathrm{GHz}$ for each band. If the band center is determined using a CMB source as seen through the atmosphere, this gives band centers $96.2,150.6$, and 208.8 for 100,150 , and $220 \mathrm{GHz}$, respectively. If the band center is determined using a typical Galactic source with dust and synchrotron emission, as seen through the atmosphere, this gives band centers 96.3, 152.4, and 212.2 for 100,150 , and $220 \mathrm{GHz}$, respectively. Another way to calculate the average band center is to compute the band center for each detector separately and then average over all the detectors in a band. Doing this for the Galactic spectrum gives average band centers of $96.3,152.3$, and $212.0 \mathrm{GHz}$ with uncertainties in the average of $0.1,0.3$, and $1.0 \mathrm{GHz}$ for 100 , 150 , and $220 \mathrm{GHz}$, respectively. The standard deviations of the distributions over the detectors are $0.4,1.4$, and $2.1 \mathrm{GHz}$ for 100,150 , and $220 \mathrm{GHz}$, respectively, showing how different the spectral responses of a given detector in a band can be. The outof-band high-frequency response is less than $-25 \mathrm{~dB}$, which was characterized by using high-pass thick-grille filters and a chopped source.

\subsection{Time Domain Data Processing}

Following the el-nod calibration and correction for spectral gain mismatch, the sum and the difference of a PSB pair are calculated. Differencing the calibrated orthogonally linearly polarized detectors removes most of the unpolarized atmospheric response and unpolarized, scan-fixed, contamination. Atmospheric $1 / f$ noise dominates the rms of the pair-sum timestreams for a typical half-scan. These fluctuations can be less than $1 \mathrm{mK}$ in good weather or reach approximately $300 \mathrm{mK}$ in bad weather, with a $1 / f$ knee as high as a few Hertz. The data also contain a sub-dominant scan-fixed contamination, which does not integrate down as uncorrelated noise. To remove both $1 / f$ atmospheric and scan-fixed contamination, the data from each half-

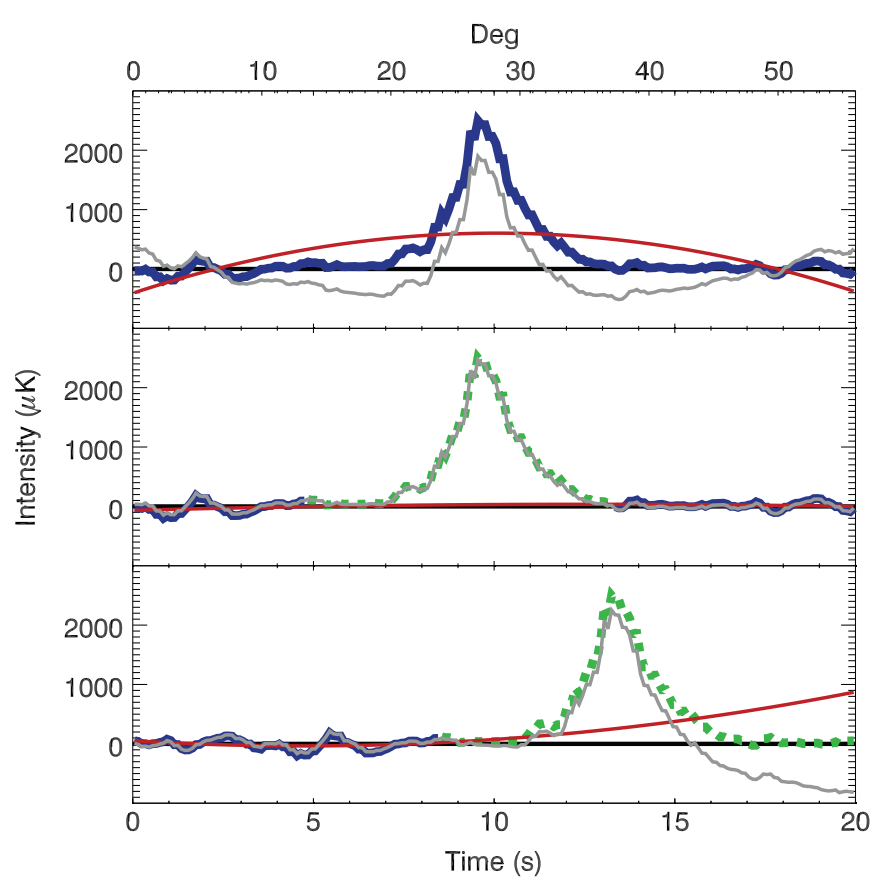

Figure 4. Detector timestream simulation showing three different polynomial filtering methods. The top plot shows a typical timestream resulting from a scan across the Galaxy (blue) with a fitted second-order polynomial (red) subtracted off, causing a significant distortion of the Galaxy (gray). The middle plot shows the same scan, except the Galaxy (green) has been excluded from the fit. The polynomial has been interpolated across the Galaxy, leading to minimal filtering effects. The bottom plot shows a typical scan that ends on the Galaxy, requiring the polynomial to be extrapolated onto the Galaxy. The extrapolated polynomial causes severe artifacts in the maps, requiring these scan portions to be excluded from the analysis.

(A color version of this figure is available in the online journal.)

scan are high-pass filtered by removing a second-order polynomial fit to each half-scan, at the cost of removing some Galactic signal as well. The pair-sum and pair-difference timestreams are treated separately but with the same filtering.

Polynomial removal causes an obvious distortion of the signal when the scans include the Galactic plane. To reduce this effect, the Galaxy is masked during the determination of the polynomial fit ("polynomial mask"). The preferred filtering scheme uses a second-order polynomial while masking out samples $|b|<4^{\circ}$. This scheme achieves a compromise between noise filtration and signal preservation. This process is not perfect and some residual filtering effects remain in the maps, as discussed in Section 3.3. A dedicated study of different filtering techniques was undertaken but none improved the maps significantly without causing worse filtering effects or adding additional noise. For example, maps with only a DC offset removed perhalf-scan not only have less filtering applied to the data but also have large-scale noise features that make quantitative analysis unreliable.

There is an added complication in this scheme for scans that end within the masked region (Figure 4). A polynomial constrained by measurements on both sides of the Galactic plane reasonably approximates the low-frequency drifts within the interpolated region. However, scans not constrained on both sides of the plane require the polynomial to be extrapolated beyond the fitted region. Extrapolated polynomials tend to diverge because there are no data constraining the fit. Therefore, the filtering scheme is modified so that scan portions that end within the masked region are excluded, giving rise to maps with 

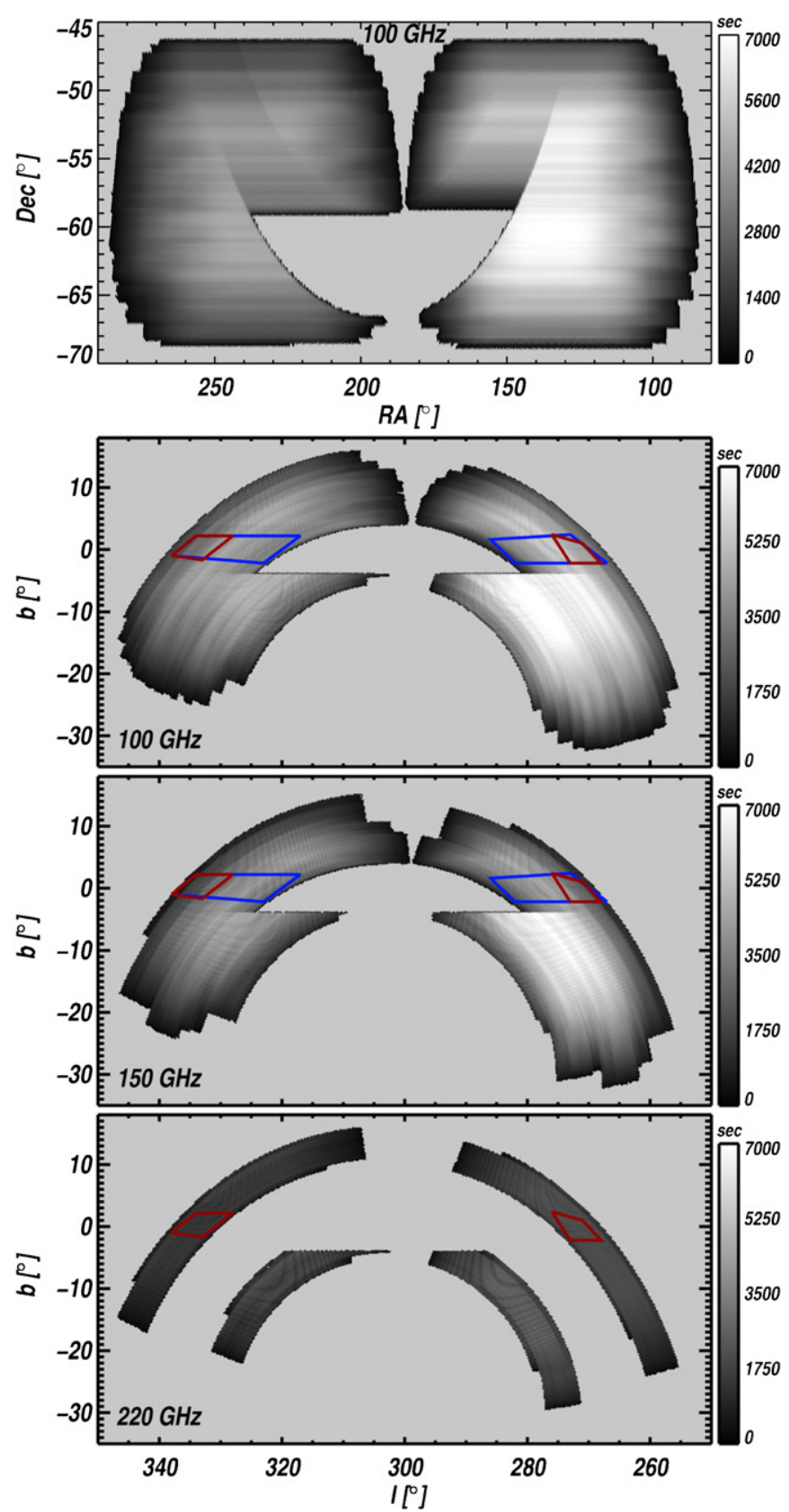

Figure 5. Integration time per $0.25 \mathrm{Healpix}$ map pixel for $100 \mathrm{GHz}$ (top Celestial coordinates, second from top: Galactic coordinates), $150 \mathrm{GHz}$ (second from bottom; Galactic coordinates), and $220 \mathrm{GHz}$ (bottom; Galactic coordinates) derived from all three seasons co-added over all four boresight angles of BICEP observations. The wedge-shaped cuts in the map without integration time are from the omission of parts of scans with extrapolated polynomial fits (Section 2.4). The $100 \mathrm{GHz}$ celestial coordinate map is an example of the native observation reference frame from the South Pole. The red outlined area (called the "220 GHz analysis region") is an area of the sky where analysis at all three bands can be carried out. This region is a subset of the blue outlined area on the 100 and $150 \mathrm{GHz}$ maps (called the "100/150 GHz analysis region") where the final analysis can only be carried out at 100 and $150 \mathrm{GHz}$ (see Section 3).

(A color version of this figure is available in the online journal.)

a missing wedge (Figure 5). Additionally, a brief measurement on both sides of the plane was not enough to constrain the polynomials sufficiently. Therefore, scans were required to have at least 10 samples on either side of the Galactic plane to be used in the mapmaking process.

\subsection{Mapmaking}

Once the data-processing steps are completed, the Stokes parameters $I, Q$, and $U$ are derived using standard techniques (Jones et al. 2007). Equation (1) can be simplified to

$$
d_{A, B}^{\prime}=g_{A, B}\left(I+\gamma_{A, B}\left(Q \cos \left(2 \psi_{A, B}\right)+U \sin \left(2 \psi_{A, B}\right)\right)\right),
$$

assuming the beam functions are the same for a given pair of PSBs and the responsivities, $g_{A, B}$, still include the spectral gain mismatch after el-nod calibration. The Stokes parameters $I, Q$, and $U$ now represent quantities integrated over $\Omega$ and $\nu$, and $\{A, B\}$ refers to the two orthogonal bolometers within a given feed.

Before calculating the sum and difference data of the two PSBs in a feed, the spectral gain mismatch factors calculated in Equation (2) are corrected:

$$
\begin{aligned}
& d_{A}=d_{A}^{\prime} \times(1+\xi), \\
& d_{b}=d_{b}^{\prime} \times(1-\xi),
\end{aligned}
$$

where $\xi$ is the spectral gain mismatch and $d_{A, B}^{\prime}$ denotes the uncorrected timestream data. The pair-sum $\left(d_{+}\right)$and pairdifference $\left(d_{-}\right)$timestreams can then be determined:

$$
\begin{aligned}
d_{+}=\frac{d_{A}+d_{B}}{2}= & I+\alpha_{+} Q+\beta_{+} U \approx I, \\
d_{-}=\frac{d_{A}-d_{B}}{2}= & \alpha_{-} Q+\beta_{-} U \approx Q \cos \left(2 \psi_{A}\right) \\
& +U \sin \left(2 \psi_{A}\right),
\end{aligned}
$$

which give rise to the Stokes parameters $I, Q$, and $U$, where $\alpha_{ \pm}$ and $\beta_{ \pm}$account for the polarization angle:

$$
\begin{aligned}
& \alpha_{ \pm} \equiv \frac{\gamma_{A} \cos \left(2 \psi_{A}\right) \pm \gamma_{B} \cos \left(2 \psi_{B}\right)}{2} \\
& \beta_{ \pm} \equiv \frac{\gamma_{A} \sin \left(2 \psi_{A}\right) \pm \gamma_{B} \sin \left(2 \psi_{B}\right)}{2}
\end{aligned}
$$

The $A$ and $B$ bolometers are assumed to be nearly perpendicular, so the pair-sum gives the total intensity to a very high precision. If $\gamma$ is also assumed to be equal to one, then $\alpha_{ \pm}$and $\beta \pm$ simplify to $\left\{0, \cos \left(2 \psi_{A}\right)\right\}$ and $\left\{0, \sin \left(2 \psi_{A}\right)\right\}$. However, $\gamma$ is closer to $90 \%$ and can differ by several percent between a given pair of bolometers within a feed, so no simplification is made during mapmaking using the pair-difference signal. After the spectral gain mismatch is corrected and after the sum and difference are taken, the resulting pair-sum and pair-difference timestreams are polynomial filtered as discussed in Section 2.4.

The $I, Q$, and $U$ maps are given by

$$
m_{(I, Q / U)}=\left(A_{T} N^{-1} A\right)^{-1} A_{T} N^{-1} d_{(+,-)},
$$

which is a noise-weighted linear least-squares regression where $m$ is the set of map pixels for a given Stokes parameter, $N$ is the noise covariance matrix, and $A$ is the pointing matrix. Since $d_{(+,-)}$are filtered timestreams, the resulting sky maps $m_{(I, Q / U)}$ are also filtered. For this work, it is sufficient to assume that the noise is uncorrelated, making $N$ diagonal and simple to invert. The variance associated with samples in a single halfscan is assumed to be time-independent, and is calculated from the samples lying outside the Galactic mask after polynomial subtraction. For the pair-sum data, the pointing matrix consists of ones and zeros, indicating whether or not the telescope is 
pointing at a particular map pixel. This simplifies Equation (11), only requiring the calculation of the weighted mean of $d_{+}$to determine the total intensity map $m_{I}$ (see Equation (5) in Chiang et al. 2010). To determine the polarization maps $m_{Q / U}$ from the pair-difference data $d_{-}$, the pointing matrix consists of a combination of the $\alpha$ 's and $\beta$ 's and Equation (11) can be written as

$$
\sum_{i=1}^{S_{j}} w_{i}\left(\begin{array}{cc}
\alpha_{i}^{2} & \alpha_{i} \beta_{i} \\
\alpha_{i} \beta_{i} & \beta_{i}^{2}
\end{array}\right)\left(\begin{array}{l}
Q_{j} \\
U_{j}
\end{array}\right)=\sum_{i=1}^{S_{j}} w_{i}\left(\begin{array}{c}
\alpha_{i} d_{i} \\
\beta_{i} d_{i}
\end{array}\right)
$$

The subscript "-_" has been dropped from the $d, \alpha$, and $\beta$ terms for clarity. Equation (12) holds true for each band separately where $d_{i}$ is a single pair-difference timestream sample, $w_{i}$ is the inverse variance for a single sample, index $j$ corresponds to a given map pixel, and $S_{j}$ is the total number of samples from all the feeds per band in a given map pixel. After summing over $i$, the matrices per pixel per band are inverted to solve for $Q$ and $U$. For each map pixel, the seven quantities used to recover $I, Q$, and $U$ are the pair-sum weights and pair-sum weighted data, pair-difference weighted data multiplied by $\alpha$ and $\beta$, and weighted $\alpha^{2}, \beta^{2}$, and $\alpha \beta$. An eighth quantity, integration time per pixel, is also recorded in order to measure noise properties and observing efficiency.

\section{RESULTS}

The BICEP polarimeter produced high signal-to-noise maps of the Galaxy in three different bands. The analysis in this section includes a qualitative discussion of the map features, investigation into map statistical and systematic uncertainties, a direct comparison to the maps observed by the WMAP satellite, and a quantitative analysis of the properties in the maps. For quantitative calculations, two analysis regions were defined (Figure 5). The "100/150 GHz analysis region" consists of 147 one-degree Healpix map pixels that have intensity values greater than zero, located at less than two degrees in Galactic latitude, and a polarization fraction magnitude less than $20 \%$. Since there were only two $220 \mathrm{GHz}$ feeds installed in BICEP, this limited the sky coverage for that band. Therefore, a " $220 \mathrm{GHz}$ analysis region," using 53 of the 147 pixels from the $100 / 150 \mathrm{GHz}$ analysis region, defines a subset of map pixels that can be analyzed at all three bands.

\subsection{Intensity and Polarization Maps}

Figures 6-9 show BICEP Galactic maps in three different bands, binned into 0.25 Healpix pixels, using second-order polynomial filtering while masking data $|b|<4^{\circ}$. Polarization angles are defined counterclockwise from the meridian at the map pixel, in accordance with the IAU definition (Weiler 1973; Hamaker \& Bregman 1996). BICEP absolute calibration casts the maps in thermodynamic temperature (Mather et al. 1994; Bennett et al. 2003), $d T$, the temperature difference (measured with respect to the mean $\mathrm{CMB}$ temperature) of a blackbody emitter required to explain the measured intensity. This unit is convenient for CMB analysis as it places the CMB anisotropy on the same scale at all observing frequencies and gives a consistent reference frame for emission from sources other than the CMB. Conversion from temperature to intensity is done via a scale factor given by the derivative of the Planck function (Equation (17)).

The total intensity maps in Figure 6 show the large contrast between the CMB temperature anisotropy $\left(|b|>5^{\circ}\right)$ and the



Figure 6. BICEP intensity maps from all three seasons co-added over all four boresight angles at 0.25 Healpix resolution, in Galactic coordinates, at 100, 150 , and $220 \mathrm{GHz}$ from top to bottom, respectively. The color scale has been chosen to emphasize the CMB anisotropy, which is visible in all three bands.

(A color version of this figure is available in the online journal.)

emission near the Galactic plane. The noise in the intensity maps is not white; however, it is barely visible at either 100 or $150 \mathrm{GHz}$, even off the plane, except at the edges of the map. The map pixels at the lower elevation have a lower signal-to-noise ratio due to the Healpix pixelization scheme that bins data into equal area pixels, while the scan strategy follows a Mercator projection. This effect is most readily visible in the $220 \mathrm{GHz}$ maps when comparing the upper and lower portions of the map.

The total intensity maps overlaid with polarization vectors in Figure 7 show that the brightest Galactic emission is within two degrees of the plane and consists of smooth large-scale features and compact sources along the plane. The maps show that the intensity signal dominates over the noise in the plane at all three bands and the polarization vectors are mostly perpendicular to the plane.

Figure 8 shows there is $U$ signal in the plane corresponding to polarization vectors that are not perfectly perpendicular to the Galactic plane. There are regions of positive $U$ polarization in the plane at all three bands. However, there is a region in the $150 \mathrm{GHz}$ maps near Galactic longitude $\ell=322^{\circ}$ that shows a significant amount of negative $U$ signal that is not an artifact of the filtering or systematics. The noise appears mostly white over the whole observing region with some residual striping along the scan direction at this map resolution. 


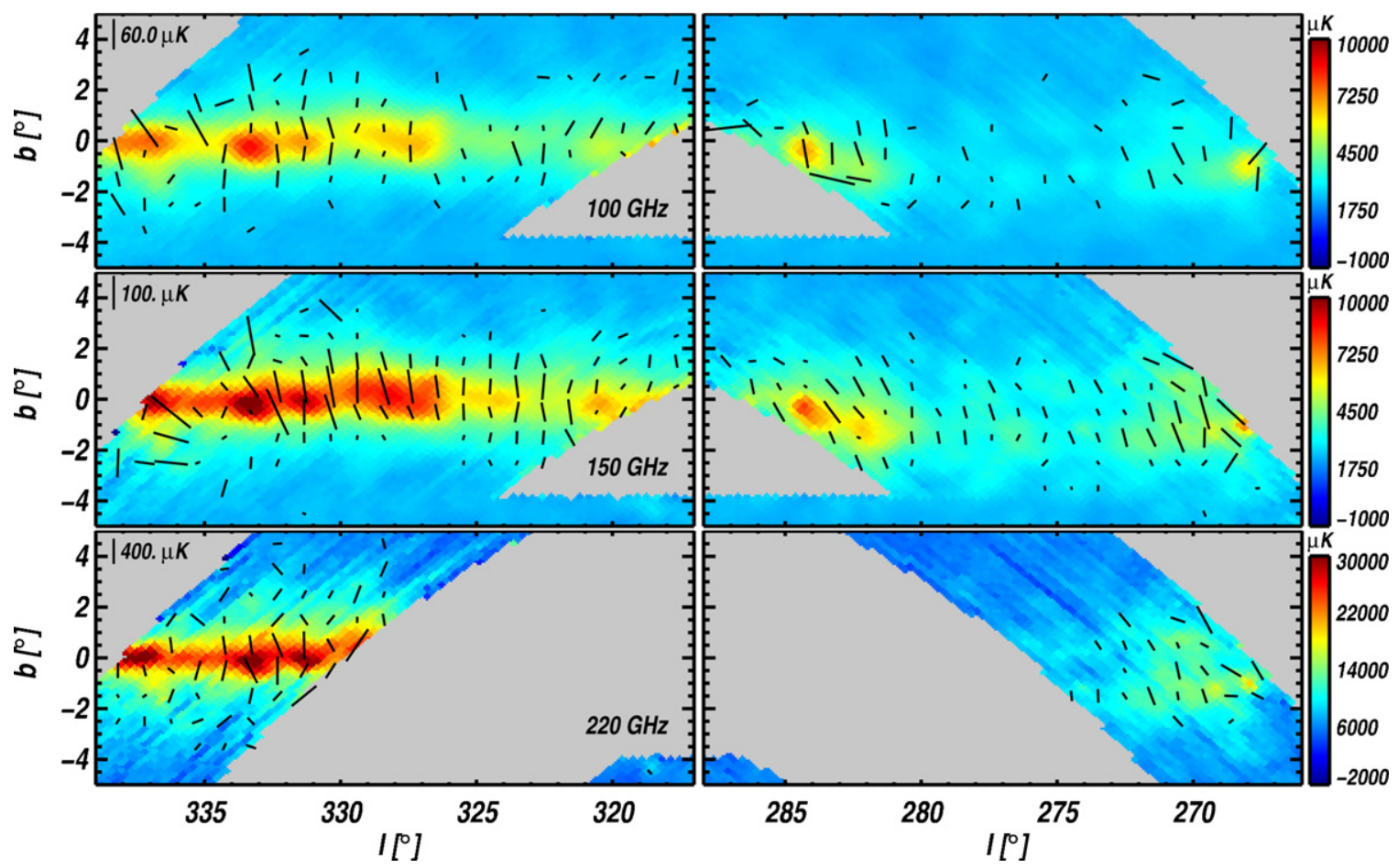

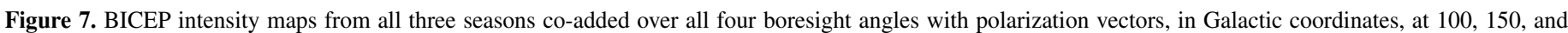

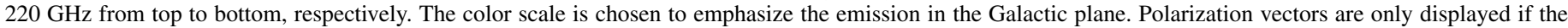

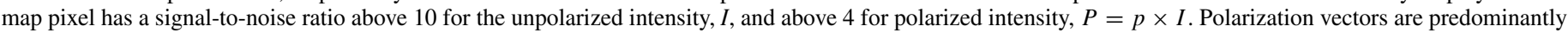



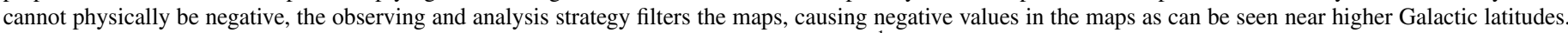

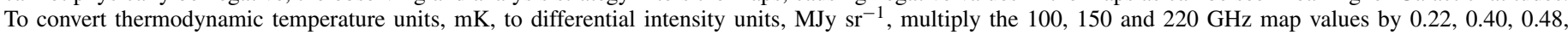
respectively.

(A color version of this figure is available in the online journal.)

Figure 9 shows there is no significant negative $Q$ polarization in the Galactic plane, which would have produced polarization vectors generally parallel to the Galactic plane. The noise is nearly identical in nature to the noise in the $U$ maps because both are derived from the same pair-difference data with identical filtering.

\subsection{Map Quantities and Polarization Fraction Model}

The map quantities studied are $I$, the pixel intensities (Section 3.5); $q$, the polarization fraction perpendicular to the Galactic plane; $\eta$, the exponent of a simple power law describing the relationship between $q$ and $I$ (Section 3.6); and $\theta$, the polarization angle in Galactic coordinates (Section 3.7). The derived variables $q, u, p$, and $\theta$ are given, respectively, by

$$
q \equiv \frac{Q}{I}, \quad u \equiv \frac{U}{I}, \quad p \equiv \sqrt{q^{2}+u^{2}}, \quad \theta \equiv \frac{1}{2} \tan ^{-1}\left(\frac{U}{Q}\right) .
$$

The main polarization quantity studied is $q$ as opposed to $p$ or $u$, because $p$ suffers noise bias being a positive definite quantity, and there is relatively little signal in $u$. While the magnitude of $u$ can make up a large fraction of the total polarization power in any given 0.25 pixel, the average $u$ power is small compared to the average $q$ power. For example, the average ratio of $u$ to $q$ at $100 \mathrm{GHz}$ in the $100 / 150 \mathrm{GHz}$ analysis region is 0.25 . Also, note that Figure 7 is at 0.25 resolution, while analysis is done at 1.0 resolution. The signal-to-noise ratio at 0.25 resolution makes it difficult to determine trends by visual inspection only, which is why the pixels are binned to $1: 0$ pixel size for analysis.
It is known that the observed polarization fraction from an astronomical source will be lessened by disorder in the magnetic field (Wiebe \& Watson 2004). To explore the Galaxy's magnetic structure, BICEP maps are fit to a phenomenological power law as

$$
q=q_{\left(I_{\text {median }}\right)} \times\left(\frac{I}{I_{\text {median }}}\right)^{\eta}
$$

where $I_{\text {median }}$ is calculated from the map pixels in the $220 \mathrm{GHz}$ analysis region, $\eta$ is the slope parameter, and $q_{\left(I_{\text {median }}\right)}$ is the overall polarization fraction normalization parameter. The parameter $\eta$ approximately represents the disorder in the magnetic field which is traced by millimeter-wave polarization.

The simplest Galactic magnetic field model to compare BICEP data to is one where $\eta=0$, which has no disorder in the field. The measured polarization is a direct imprint of the intensity signal, related by a constant polarization factor $q_{0}$.

Other models describe the Galaxy's magnetic fields as being much more random. For example, a simple toy model for this case is one where the Galaxy is uniformly thick and consists of a constant polarized $\left(q_{0}\right)$ diffuse component emitting with a weak intensity, $I_{0}$. Scattered throughout are dense star-forming regions with random polarization angles which, when integrated along the line of sight or over the beam width, will integrate down to very low net polarization but will contribute to total intensity with $I_{1}$. In this case, the polarization fraction would be

$$
q=\frac{q_{0} I_{0}}{I_{0}+I_{1}} \propto I^{-1}
$$



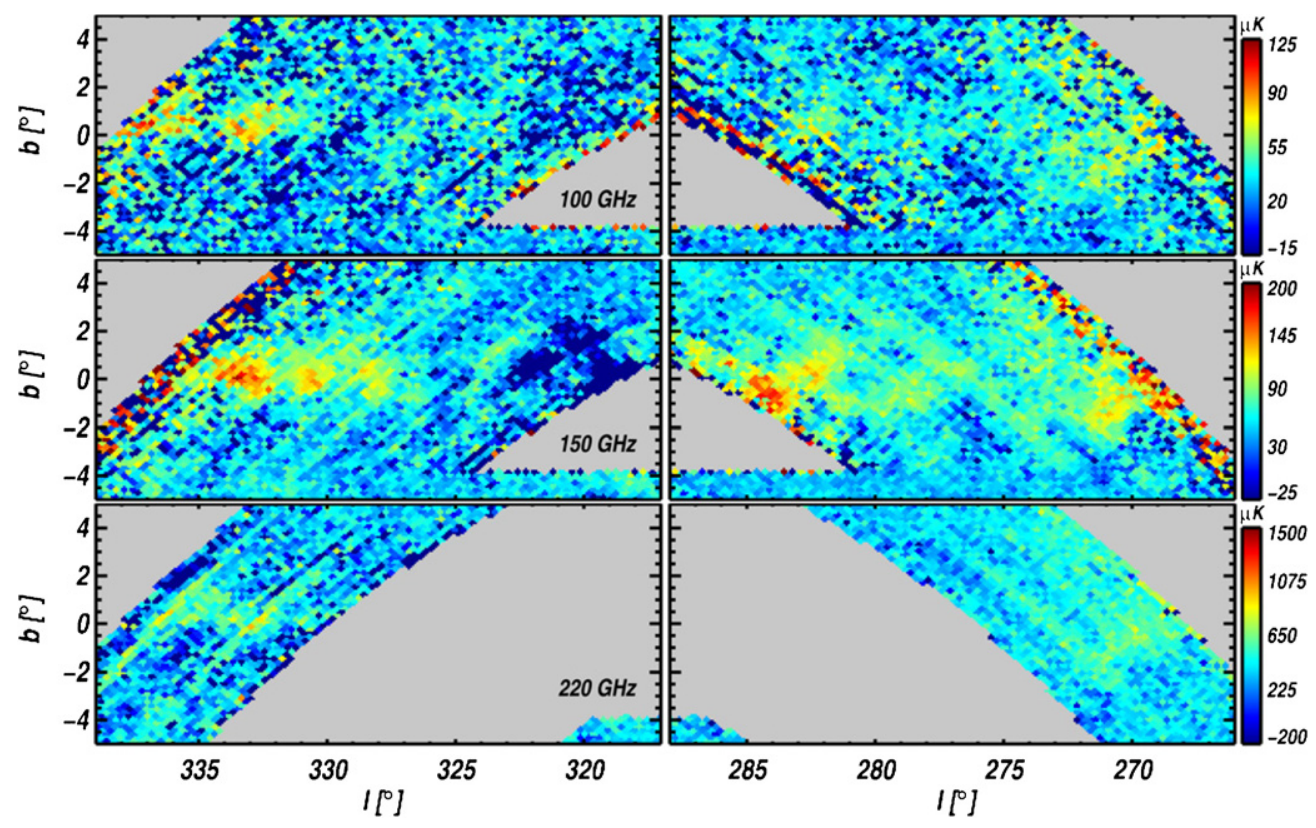

Figure 8. BICEP $U$-polarization maps from all three seasons co-added over all four boresight angles, in Galactic coordinates, at 100,150 , and $220 \mathrm{GHz}$, from top to bottom, respectively. Galactic $+U$ polarization corresponds to a polarization vector in the $+b$ and $+\ell$ directions. Power can be seen in all three bands indicating areas where the Galactic magnetic field is not exactly aligned with the Galactic plane. The significant detection of negative $U$ power in $150 \mathrm{GHz}$ map at Galactic longitude $322^{\circ}$ is physical and not an artifact of filtering or other systematics.

(A color version of this figure is available in the online journal.)
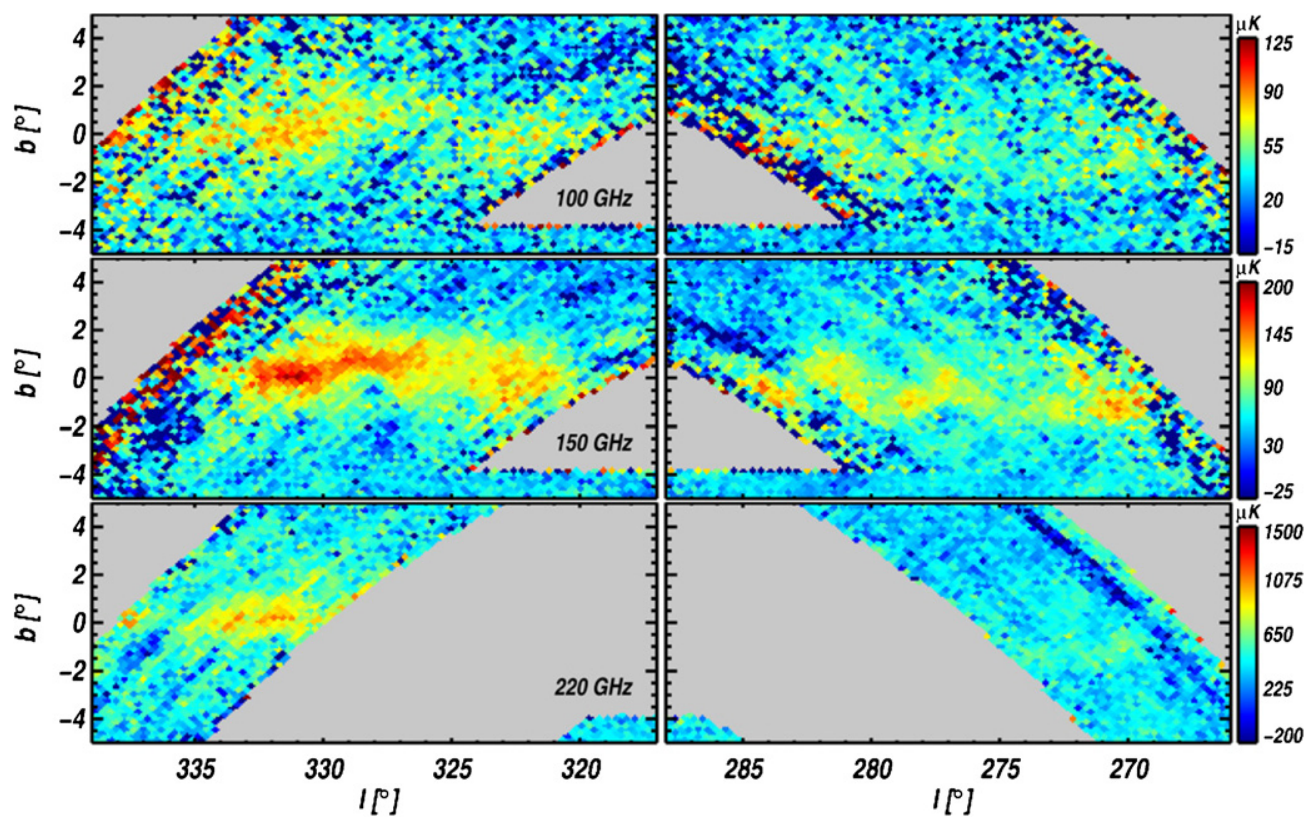

Figure 9. BICEP $Q$-polarization maps from all three seasons co-added over all four boresight angles, in Galactic coordinates, at 100, 150, and $220 \mathrm{GHz}$, from top to bottom, respectively. Galactic $+Q$ polarization (red), corresponding to a vector that is perpendicular to the Galactic plane, dominates the maps.

(A color version of this figure is available in the online journal.)

In this case $\eta=-1$, implying increasing dust column density that contributes no additional polarized intensity.

While both of the models here are strictly empirical, other studies have used similar methods. These methods involve fitting starlight polarization to a similar power-law model; the exponent for polarization by absorption is related to the power-law exponent for polarization by emission in the millimeter-wave band by $\eta_{\mathrm{em}}=\eta_{\mathrm{abs}}-1$. Fosalba et al. (2002) fit starlight polarization data to $p$ versus $E(B-V)^{\eta_{\text {abs }}}$, finding $\eta_{\text {abs }}=0.8$, which implies $\eta_{\mathrm{em}}=-0.2$. Fosalba et al. (2002) then relate this fit parameter to a magnetic field model from Burn (1966) using the assumption above, namely that the polarization fraction is a product of the ratio of uniform and random components of the magnetic field. Jones (1989) fits starlight polarization data to $p$ versus $\tau_{K}^{\eta_{\text {abs }}}$, finding $\eta_{\mathrm{abs}}=0.75$, which implies $\eta_{\mathrm{em}}=-0.25$. Jones (1989) takes the fitting process a step further and runs Monte Carlo simulations for observing Galactic magnetic field arrangements with varying level of randomness and claims a more accurate fit than using an analytic equation. Lastly, these other works study polarization fraction $p$, while in this paper $q$ is studied, 



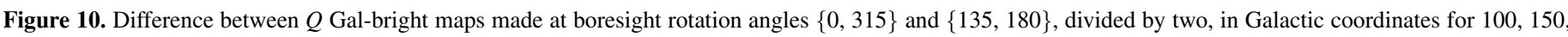



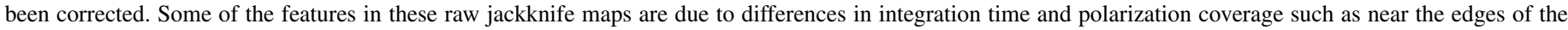

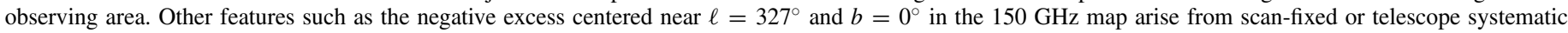

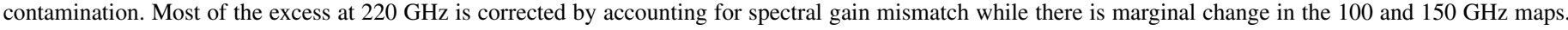
There is some faint striping nearly orthogonal to the Galactic plane in the 150 and $220 \mathrm{GHz}$ maps from residual $1 / f$ noise leaking through the polynomial mask.

(A color version of this figure is available in the online journal.)

which does not necessarily follow the same power-law trend as $p$. For example, a Galaxy with completely random magnetic field directions would predict a polarization fraction $p$ given by a power-law exponent of $\eta=-0.5$; however, in this scenario, $q$ would oscillate about zero and give an average $\eta=0$.

\subsection{Noise and Systematic Error Evaluation}

To confirm the integrity of the maps presented here, they were cross-checked with an independently written pipeline. The two pipelines produced nearly identical Healpix maps, reducing the possibility of coding errors or other non-physical errors. For example, taking the difference between the two pipeline's $150 \mathrm{GHz} Q$ maps gave a pixel rms level five times smaller than the noise level, with no obvious signal features left.

The data have $1 / f$ noise from atmospheric fluctuations, electronic readout drifts, thermal instabilities, and scan-fixed contamination. The polynomial subtraction removes most of this contamination but the polynomial masking allows some of this noise to leak back into the Galaxy maps. The polynomial fitting is applied only to data outside of the Galactic plane mask, causing $\chi^{2}$ not to be minimized with respect to the noise within the mask. However, since the noise is correlated, the polynomial fit approximates well the noise close to the mask edges, but decreases in effectiveness the further the pixels are from the edge of the mask. Therefore, the larger the mask used, the worse the polynomial fit inside the mask approximates the noise, because there is a larger gap over which the polynomial must be interpolated. The smaller the mask used, the better the fit approximates the noise; however, this also increases the filtering of the signal in the plane.

Optical imperfections, such as beam mismatch, crosspolarization, depolarization, and sidelobe response can cause systematic changes in the maps. These "telescope systematics," studied and characterized by Takahashi et al. (2010) for the CMB B-mode analysis, are controlled very well partly because of BICEP's simple, compact design. The overall magnitude of the telescope systematics and $1 / f$ leakage in the Galactic maps are estimated by splitting the data into two halves, according to the telescope's orientation angle about the boresight. Angles $0^{\circ}$ and $315^{\circ}$ are called "boresight map A," while $135^{\circ}$ and $180^{\circ}$ are called "boresight map B." Since there are only two $220 \mathrm{GHz}$ feeds, $220 \mathrm{GHz}$ boresight map A is determined from a single feed, while $220 \mathrm{GHz}$ boresight map B is determined from the other feed. This split is the most probative for the polarimeter because the Galactic sky coverage in each half comes mostly from a different set of feeds, taken at different times, under different weather conditions, and with the telescope oriented differently with respect to gravity. For this study, the two BICEP boresight maps per frequency band are studied in this paper, which can be used to gauge the general level of residual systematic contamination in the maps.

\subsubsection{Boresight Difference Maps}

To illustrate the efficacy of the boresight difference maps, Figure 10 shows the difference between the two groups for the $Q$ maps for all three bands, both before and after the spectral gain mismatch correction. This is a qualitative jackknife test, as it does not test quantitatively against the expected noise or signal leakage. Figure 10 shows some power in the $Q$ boresight map jackknife at all three bands, which is representative of all the Galactic jackknife maps. The residual power in the $220 \mathrm{GHz}$ raw maps was used to validate the spectral gain mismatch model and the post-corrected maps show the level of correction achieved. While there is still some residual power in the 100 and $150 \mathrm{GHz}$ channels, it does not affect the results claimed in 
this paper. The post-corrected $220 \mathrm{GHz}$ maps give consistent polarization results between the two detectors; however, there is still uncertainty in the spectral gain mismatch parameter mostly due to variability in atmospheric conditions.

\subsubsection{Uncertainty due to Spectral Gain Mismatch}

Simulations were run to show the level of uncertainty induced on the quantitative parameters analyzed in this paper due to uncertainty in the spectral gain mismatch calculation. Two sets of simulations were run; one based on the uncertainty in the atmospheric conditions and one based on the uncertainty in the measured spectral response.

The average spectral response and measurement uncertainty were calculated from the eight separate spectral response runs per detector (Section 2.3). The uncertainty in atmospheric conditions was derived from computing the spectral gain mismatch using Austral winter and summer conditions, using a low precipitable water vapor (pwv) value, a median value, and a high value for different observing angles. The mean and standard deviation of these conditions were computed, where the mean values are the nominal spectral gain mismatch and the standard deviation is used as the uncertainty. The pwv conditions used were quite conservative and actual observing conditions most likely had a smaller range of weather conditions.

Simulations were performed by generating random spectral gain mismatch parameters for each feed, creating a modified list of spectral gain mismatches. Using the modified list, the entire set of mapmaking steps of Sections 2.4 and 2.5 are repeated, and $q_{\left(I_{\text {median }}\right)}$ and $\eta$ are found. This procedure was repeated ten times for both the atmospheric and spectral response measurement uncertainties, giving average parameter values and an uncertainty on each average.

The percent error computed from the simulations based on different atmospheric conditions is $\frac{\delta q_{\left(I_{\text {median }}\right)}}{q_{\left(I_{\text {median }}\right)}}=[1.0 \%, 1.3 \%$, $3.6 \%]$ and $\frac{\delta \eta}{\eta}=[8.9 \%, 15 . \%,>100 \%]$ for 100,150 , and $220 \mathrm{GHz}$ bands, respectively. The uncertainty from spectral measurement errors is $\frac{\delta q_{\left(I_{\text {median }}\right)}}{q_{\left(I_{\text {median }}\right)}}=[0.2 \%, 0.2 \%, 0.3 \%]$ and $\frac{\delta \eta}{\eta}=[2.4 \%, 1.8 \%, 41.0 \%]$ for 100,150 , and $220 \mathrm{GHz}$ bands, respectively. The uncertainty on the $220 \mathrm{GHz}$ parameters is larger than the other two bands because the two $220 \mathrm{GHz}$ feeds have the largest spectral gain mismatch and the smallest measured $\eta$ value. The spectral measurement uncertainties are five to ten times smaller than the uncertainties from the various weather conditions. The combined spectral gain mismatch uncertainties are relatively minor for the determination of $q_{\left(I_{\text {median }}\right)}$; however, these uncertainties provide the largest single source of error on $\eta$ at all three bands.

\subsubsection{Uncertainty due to Polynomial Filtering}

Polynomial subtraction removes signal as well as noise, causing systematic filtering effects. To estimate the filtering effects, simulations are carried out to compare maps before and after various filtering strategies as well as to compare to the real data. The general algorithm for the simulation was to use an intensity map and Galactic polarization model parameters to generate $I, Q$, and $U$ maps. These maps are then filtered in various ways and compared to the original maps and actual data filtered in a similar manner to find the resulting effects and uncertainties of filtering on the maps.

The simulations used WMAP $94 \mathrm{GHz}$ DA 1 (Section 3.4) and FDS (Finkbeiner et al. 1999) to make two separate intensity maps per frequency band. The WMAP intensity map was first deconvolved with the beam function provided by WMAP and convolved with the BICEP Gaussian beam at a given band. Then, the maps were downsampled from $0.125 \mathrm{Healp}$ ix pixels to 0.25 pixels. An additional simulation was carried out using the higher resolution pixels, but this caused less than a 5\% change in the resulting simulation values. For the FDS simulation, tools are provided to predict the power over the entire sky at an arbitrary frequency. ${ }^{16} \mathrm{~A}$ full-sky prediction is made at 0.016 Healpix pixel resolution for each BICEP band for each measured spectral bandpass data point (approximately 200 separate frequency points per band), which can then be summed together to make three full-sky-simulated BICEP band dust maps. The maps are convolved with the BICEP beam at each band and downsampled to 0.25 Healpix resolution.

Using these intensity maps, simulated polarization maps are generated using Equation (14) with parameters found from the BICEP maps. For the simulations, the polarization fraction magnitude was always chosen to be $2 \%$ at the median intensity and the power-law exponent $\eta$ was varied between -0.75 , -0.50 , and -0.25 . The polarization angle is set to $7^{\circ}$, the approximate average polarization angle found in the $220 \mathrm{GHz}$ analysis region, which sets the value of $U$ in the simulation. Using this model, the polarization fraction has the possibility of taking unphysical values; therefore, the maximum polarization fraction allowed is set to $10 \%$. A value of $25 \%$ was also implemented but this did not change the results appreciably.

BICEP pointing was used to extract $I, Q, U$ values from the simulated maps to generate the simulated timestreams. The simulated timestreams were processed with the same filtering as described in Section 2.4, leading to identical coverage as the real data. In addition to the nominal $4^{\circ}$ polynomial mask, separate simulations were performed using $2^{\circ}$ to $9^{\circ}$ polynomial masks for the $W M A P$ simulations. Mask values above $5^{\circ}$ gave marginal improvements in recovery of studied parameters but these higher mask values also decreased the amount of mapped area and also created more map pixels near the edges of the observation area with abnormal properties. Polynomial masks ranging from $2^{\circ}$ to $5^{\circ}$ were also applied to the real data. Then, for each map, the same pixels were fit to Equation (14) and the average angle and standard error in the angle were computed.

Figure 11 shows the results of the WMAP simulation (FDS simulation results not shown) and compares the resulting average map parameters to the real data. The changes in derived parameters due to polynomial-mask size are generally smaller than the changes in the parameters between filtered and unfiltered maps. The level of filtering effects on the maps and uncertainty in the map correction factor can be judged from the different simulations and the comparison to the real data. The filtering effects and correction factors are smaller and better understood for some parameters such as the intensity $I$, but are larger and not as well understood such as for $\eta$. The conclusion from the simulations and comparison to the real data in Figure 11 is that while the BICEP maps have been systematically filtered, the magnitude of this effect is small enough not to affect the general results from the analysis of the maps. However, these effects do prevent additional quantitative analysis of the maps beyond what is performed in this paper as a whole.

The uncertainty in the filtering correction for the intensity data is $\pm 5 \%$, which comes mostly from the variation of results from the WMAP and FDS simulations rather than variations within

\footnotetext{
16 http://astro.berkeley.edu/dust/index.html
} 

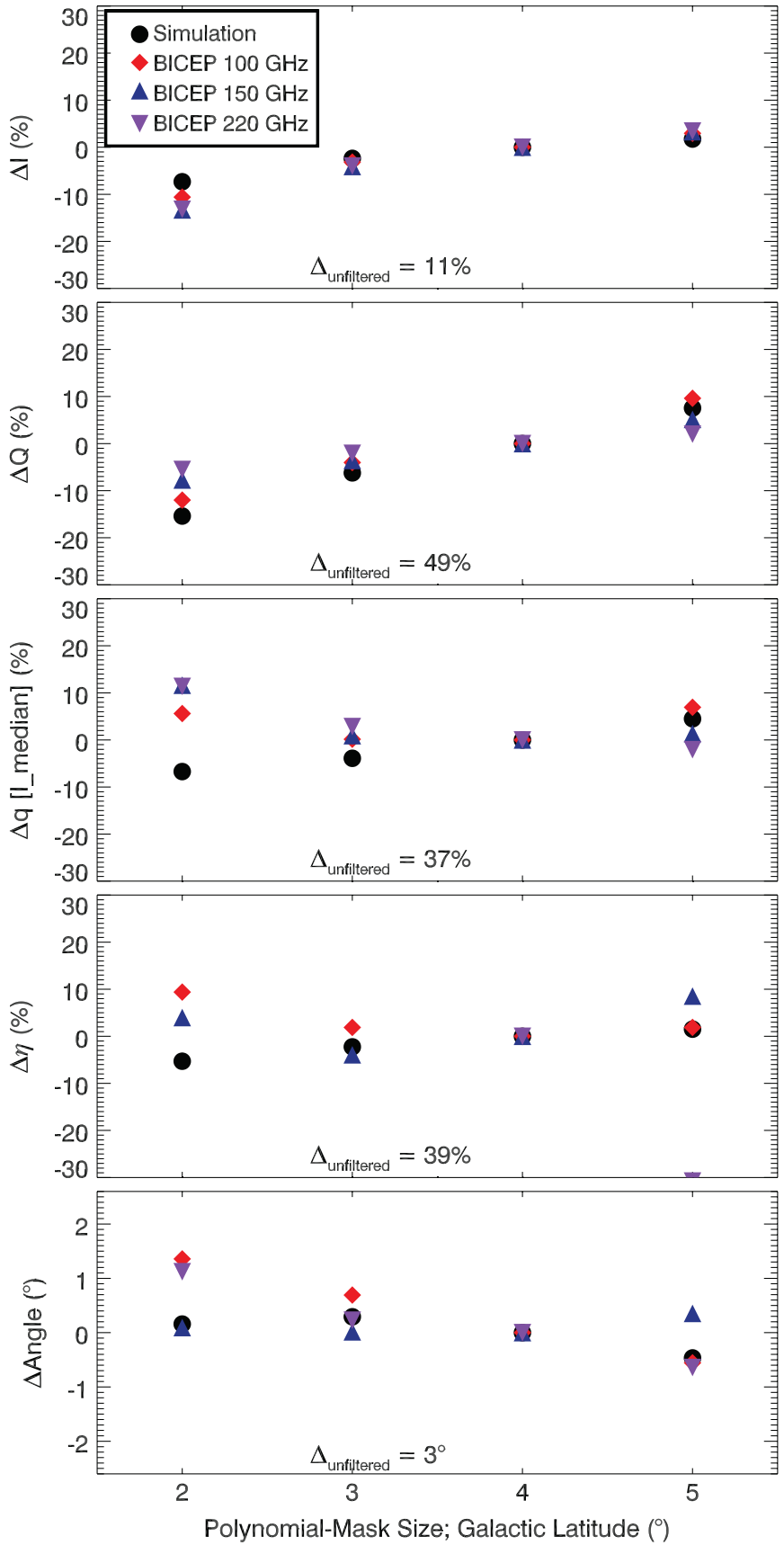

Figure 11. Fractional change in the average map parameters vs. polynomialmask size for $100 \mathrm{GHz}$ (red diamonds), $150 \mathrm{GHz}$ (blue triangles), $220 \mathrm{GHz}$ (purple upside-down triangles), and the $150 \mathrm{GHz} W M A P$ simulation (black circles) showing the size of, and general level of uncertainty in, the filtering effects on the maps. The parameters studied are intensity $I, Q$ polarization, $Q$ polarization fraction at the median intensity, $\eta$, and polarization angle. The units are relative percent difference (angular difference for the bottom plot) between a given average map parameter, $X$, and the $4^{\circ}$ polynomial-mask average map parameter, $X_{4^{\circ}} ; \Delta X=\left(X / X_{4^{\circ}}-1\right)$. At the bottom of each plot is the fractional difference, $\Delta_{\text {unfiltered }}$, in the parameter value derived from the average values of unfiltered simulation maps compared to that from filtered simulation maps with a $4^{\circ}$ polynomial mask.

(A color version of this figure is available in the online journal.)

the simulation parameters. The uncertainty in the polarization filtering correction is only known to $\pm 25 \%$, which comes equally from variations of simulation polarization parameters across each band and from the difference between WMAP and FDS simulations. The average correction factors for $I, q_{\left(I_{\text {median }}\right)}$,

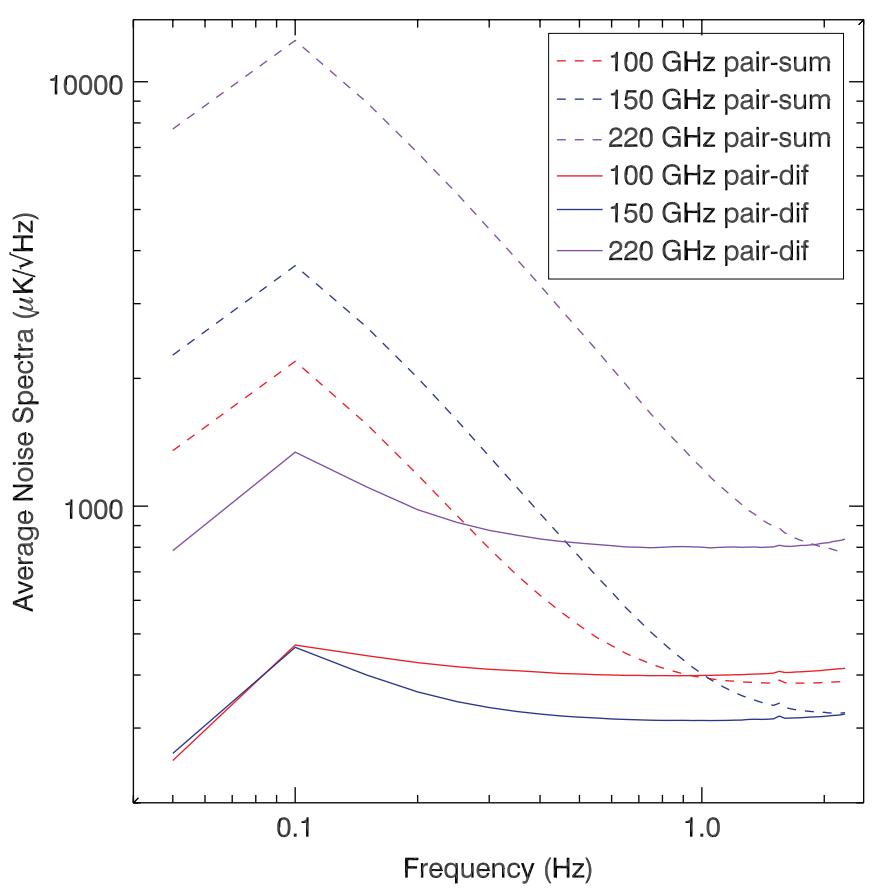

Figure 12. Average periodogram for the pair-sum and pair-difference timestreams from all of the Galactic scans, after second-order polynomial removal. The second-order polynomial filtering is apparent at $0.05 \mathrm{~Hz}$ (the lowest frequency bin), where the power is lower than the white-noise level. The rise at high frequency is due to the deconvolution of the detector and system time constant.

(A color version of this figure is available in the online journal.)

and $\eta$ calculated from these simulations for the $220 \mathrm{GHz}$ analysis region are $11 \%, 37 \%$, and $39 \%$, respectively. The average correction factor for computing the average $q$ is $23 \%$. These values are used to correct results for further analysis in this paper.

\subsubsection{General Map Noise}

Background photon and detector noise dominates the whitenoise floor in the maps. The average polarization map pixel sensitivity can be calculated by taking the calibrated timestreams and computing the periodogram for each half-scan. The average periodogram per feed for each frequency band is then shown in Figure 12. The pair-sum data suffer from increasing levels of $1 / f$ atmospheric contamination from $100 \mathrm{GHz}$ to $220 \mathrm{GHz}$. Most of the $1 / f$ atmospheric noise is removed from the polarization data by pair differencing within a feed, resulting in nearly white noise at all three bands above $0.1 \mathrm{~Hz}$. Averaging the pair-difference periodogram from $0.1 \mathrm{~Hz}$ to $1.0 \mathrm{~Hz}$ (corresponding to an angular size of $\approx 0.5-5^{\circ}$ ) gives noise-equivalent temperature (NET) per detector values of 520,450 , and $1040 \mu \mathrm{K} \sqrt{s}$ for 100,150 , and $220 \mathrm{GHz}$, respectively. After accounting for polarization efficiencies, these correspond to NEQ per feed values of 410, 340 , and $880 \mu \mathrm{K} \sqrt{s}$ for 100,150 , and $220 \mathrm{GHz}$, respectively. There is very good agreement between the noise estimate from the periodograms in Takahashi et al. (2010), which used two years of third-order polynomial filtered data from the CMB region, and this calculation using three years of data from the Gal-bright and Gal-weak regions that has second-order polynomial filtering.

The noise in a given map pixel is calculated using the NEQ per feed values and the integration time per $1^{\circ}$ pixel, assuming the integration time is split evenly between the $Q$ and $U$ maps. The 
average sensitivity for $Q$ or $U$ map pixels in the $220 \mathrm{GHz}$ analysis region is $2.8,2.8,16.0 \mu \mathrm{K}-\mathrm{rms}$ for 100,150 , and $220 \mathrm{GHz}$, respectively.

Another method to gauge the noise in the maps is to split the data in half and take the difference, which cancels the signal and leaves the residual noise. Maps were made from right-going and left-going scans separately, and then differenced. This split is used to test for detector time constant mismatch or general telescope thermal effects; however, it tends to be one of the least probative for BICEP because the two halves are taken at nearly the same time, under the same weather conditions, and with the same set of feeds. Pixels from the $Q$ and $U$ differenced maps, with a significant amount of integration time, were multiplied by the square root of integration time per pixel and the standard deviation of all the pixels was computed. This was done for all the pixels and the $220 \mathrm{GHz}$ analysis region pixels. Using all the pixels, the average noise values are 420,320 , and $930 \mu \mathrm{K} \sqrt{s}$ for 100,150 , and $220 \mathrm{GHz}$, respectively, which are comparable to the NEQ per feed noise estimate from the periodogram method, indicating good agreement between the two methods. The noise for the $220 \mathrm{GHz}$ analysis region pixels as compared to the entire map is $20 \%, 40 \%$, and $45 \%$ higher for 100,150 , and $220 \mathrm{GHz}$, respectively, due to excess $1 / f$ noise leaking inside the mask.

\subsection{WMAP $94 \mathrm{GHz}$ Band Comparison}

WMAP is a millimeter-wave satellite that has 10 differencing assemblies (DAs), all producing $I, Q$, and $U$ maps over the whole sky using one channel at $23 \mathrm{GHz}$, one at $33 \mathrm{GHz}$, two at $41 \mathrm{GHz}$, two at $61 \mathrm{GHz}$, and four at $94 \mathrm{GHz}$. The combination of BICEP data with WMAP data provides frequency coverage in the $220 \mathrm{GHz}$ analysis region from 23 to $220 \mathrm{GHz}$. As an important cross-check on the validity of the BICEP observations, a direct comparison is made between the WMAP $94 \mathrm{GHz}$ band and BICEP $100 \mathrm{GHz}$ intensity maps.

There are two systematic differences that prevent direct comparison. First, the BICEP beam has a different size than the WMAP beam and the maps are filtered as described in Section 3.2. Therefore, to make a direct comparison, each WMAP DA map is smoothed to BICEP's beam resolution, sampled into BICEP-like timestreams, and filtered. For consistency, sampling of the WMAP maps was performed using both 0.125 maps, and the nominal 0.25 maps. Since both sets of maps are filtered, there is no need for systematic filtering correction in this case.

Second, there is a difference in spectral bandpass between the two experiments. BICEP is calibrated to WMAP by comparing the CMB fluctuations in the low astronomical foreground region. However, Galactic emission has a different spectrum than the $\mathrm{CMB}$, and combined with the different bandpass response, this could cause a systematic difference between the two experiments. In a manner very similar to the spectral gain mismatch calculation in Section 2.3, the expected miscalibration is calculated using the average BICEP $100 \mathrm{GHz}$ spectral response, the average WMAP $94 \mathrm{GHz}$ band spectral response, the CMB anisotropy spectrum as seen from space and from the South Pole, and the typical Galactic source spectrum as seen from space and the South Pole.

Different atmospheric observing conditions were simulated for BICEP (Section 2.3) to compare to WMAP, which is in space and is not affected by a change in atmospheric conditions. The mean and standard deviation of spectral gain mismatch from the different atmospheric conditions between BICEP $100 \mathrm{GHz}$ and WMAP $94 \mathrm{GHz}$ band is $0.322 \% \pm 0.001 \%$ (the positive sign indicates an increase in BICEP power relative to $W M A P)$. The smallness of the difference results from the slope of the emission spectra being nearly identical for the CMB and the typical Galactic source spectrum at this particular band (this is not true at other bands), so even though BICEP and $W M A P$ have relatively different bandpasses, there is no spectral gain mismatch between the experiments. The extremely small standard deviation on this quantity results from the insensitivity of the BICEP $100 \mathrm{GHz}$ band to different atmospheric conditions, especially the emission lines outside the band. Instead of using the average $100 \mathrm{GHz}$ spectral response, this calculation was repeated for each individual detector's spectral response, giving an average and standard deviation of anomalous gain factors of $0.315 \% \pm 0.055 \%$, consistent with the first method.

The data from the two experiments were compared in a manner similar to the absolute calibration routine from Chiang et al. (2010), except the comparison was performed using maps (called "MAP") instead of spherical harmonic transform coefficients $\left(a_{\ell m}\right)$. The anomalous gain factor comparing the BICEP $100 \mathrm{GHz}$ and WMAP $94 \mathrm{GHz}$ band intensity maps is calculated as

$$
g_{\mathrm{ijk}}^{\text {anom }}=\frac{\left\langle\mathrm{MAP}_{\mathrm{WMAP}_{j}} \times \mathrm{MAP}_{\mathrm{BICEP}_{i}}\right\rangle}{\left\langle\mathrm{MAP}_{\mathrm{WMAP}_{j}} \times \mathrm{MAP}_{\mathrm{WMAP}_{k}}\right\rangle}-1,
$$

where the angle brackets represent a weighted average of the $220 \mathrm{GHz}$ analysis region pixels using the BICEP integration time as the weights. The index ( $i$ ) for BICEP corresponds to the two boresight maps (Section 3.3.1) and the indices $(j, k)$ for $W M A P$ correspond to the four DAs at $94 \mathrm{GHz}$ where $j \neq k$, so the noise in a given map is not rectified. As a consistency check on this method, the gain was computed for the CMB observing region and compared to the absolute calibration numbers from Chiang et al. (2010), who found the gains using the angular power spectra gave consistent results. The two boresight maps give values of $5.0 \%$ and $4.7 \%$ gain increases using the 0.25 maps and $4.2 \%$ and $4.0 \%$ gain increases using the 0.125 maps. Each has a $0.5 \%$ statistical error derived from using the different combinations of WMAP $94 \mathrm{GHz}$ band DA maps.

To check the uncertainty based on pixel selection or sky variance, a comparison was done using all pixel values $|b|<3^{\circ}$ in the BICEP observing region (335 one-degree pixels) for the 0.125 maps. For each of 1000 different trials, 53 pixels were chosen at random and the anomalous gain was computed. The mean and standard deviation of the distribution gave $3.1 \% \pm$ $2.1 \%$ and $2.5 \% \pm 1.9 \%$ for the two boresight maps. For comparison, the same procedure was repeated for the 253 onedegree pixels between $-3^{\circ}$ and $-6^{\circ}$ in Galactic latitude, giving anomalous gain values of $1.0 \% \pm 8.5 \%$ and $-5.0 \% \pm 6.5 \%$.

Chiang et al. (2010) quote the absolute gain uncertainty to be $2 \%$ for the BICEP maps, which decreases the relative significance of the anomalous gain factor found here. The difference in the anomalous gain factor due to the bandpass differences was calculated to be a very small; however, this calculation used a simple two-component continuum Galactic spectrum. The real Galactic spectrum is undoubtedly more complicated, which could lead to a larger spectral factor difference. Another likely cause of anomalous gain is from systematic uncertainties in the processing of raw to BICEP-filtered WMAP maps (Section 3.2), either from the beam correction or flat interpolation procedure. Regardless of the underlying cause, the $4 \%$ gain difference does not represent a significant detection of a deviation between the two experiments in the Galaxy. A larger difference was found between WMAP and QUaD (Culverhouse et al. 2010), despite 
Table 2

Raw Fit Parameters for $q$ versus $I$

\begin{tabular}{|c|c|c|c|c|c|c|c|c|c|}
\hline Experiment Channel & $\begin{array}{l}\text { Frequency } \\
\quad(\mathrm{GHz})\end{array}$ & $\begin{array}{c}I_{\text {median }} \\
(\mu \mathrm{K})\end{array}$ & $\begin{array}{c}I_{\text {median }} \\
\left(\mathrm{MJy} \mathrm{sr}^{-1}\right)\end{array}$ & $\begin{array}{c}q_{\left(I_{\text {median }}\right)} \\
(\%)\end{array}$ & $\begin{array}{l}\Delta q \\
(\%)\end{array}$ & $\eta$ & $\Delta \eta_{\text {stat }}$ & $\Delta \eta_{\text {sysA }}$ & $\Delta \eta_{\mathrm{sysB}}$ \\
\hline$W M A P \mathrm{~K}$ & 23 & 8500 & 0.14 & 1.5 & 0.10 & -0.36 & 0.05 & $\mathrm{~N} / \mathrm{A}$ & $\mathrm{N} / \mathrm{A}$ \\
\hline$W M A P \mathrm{Ka}$ & 33 & 3400 & 0.11 & 1.3 & 0.10 & -0.45 & 0.06 & $\mathrm{~N} / \mathrm{A}$ & $\mathrm{N} / \mathrm{A}$ \\
\hline$W M A P \mathrm{Q}$ & 41 & 1900 & 0.093 & 1.3 & 0.11 & -0.48 & 0.06 & 0.04 & $\mathrm{~N} / \mathrm{A}$ \\
\hline$W M A P \mathrm{~V}$ & 61 & 880 & 0.088 & 1.7 & 0.17 & -0.56 & 0.08 & 0.02 & $\mathrm{~N} / \mathrm{A}$ \\
\hline WMAP W & 94 & 800 & 0.18 & 2.8 & 0.27 & -0.60 & 0.10 & 0.06 & $\mathrm{~N} / \mathrm{A}$ \\
\hline BICEP 100 & 95.5 & 800 & 0.18 & 2.5 & 0.16 & -0.64 & 0.09 & 0.10 & 0.041 \\
\hline BICEP 150 & 149.8 & 1400 & 0.56 & 3.4 & 0.20 & -0.56 & 0.10 & 0.07 & 0.063 \\
\hline BICEP 220 & 208.2 & 4400 & 2.1 & 2.8 & 0.40 & -0.08 & 0.29 & 0.23 & 0.24 \\
\hline
\end{tabular}

Notes. Average fit parameters and errors for Equation (14) using map pixels from WMAP and BICEP in the $100 / 150 \mathrm{GHz}$ analysis region (except for $220 \mathrm{GHz}$ which uses pixels from the $220 \mathrm{GHz}$ analysis region). The BICEP fit parameters have been corrected for filtering effects (Section 3.3.3). The statistical errors for both BICEP and WMAP come from the nonlinear fits, while the systematic errors "A" for BICEP come from the two boresight maps and for WMAP, the different DAs. The systematic errors "B" for the BICEP $\eta$ parameters come from atmospheric uncertainty on spectral gain mismatch (Section 2.3). The median intensities are set by taking the median value within the analysis region (53 pixels) at a given band and rounded to two significant figures.

the similarity between the BICEP and QUaD instruments and analysis approach. However, QUaD's high-frequency cutoff is higher and low-frequency cutoff lower than either BICEP or $W M A P$, making it sensitive to certain emission lines to which BICEP and WMAP are insensitive.

\subsection{Intensity vs. Frequency}

By studying the spectrum of the unpolarized and polarized emission for a given point on the sky, it is possible to understand the composition of the ISM across the sky. The spectral response plots of intensity versus frequency can show the fraction of dust and synchrotron in the Galactic plane. WMAP provides a vast amount of information on this topic; however, their highest frequency band still has an appreciable amount of synchrotron emission. Above $150 \mathrm{GHz}$, most Galactic emission comes from dust, which is where BICEP's 150 and $220 \mathrm{GHz}$ channels add unique information.

A common unit for distributed astrophysical millimeter-wave emission is differential intensity as opposed to thermodynamic temperature, where the conversion factor from thermodynamic units can be calculated as

$$
d I=\left.\frac{d B}{d T}\right|_{2.725 \mathrm{~K}} \times d T,
$$

and $B$ is the Planck blackbody function. The derivative with respect to temperature is

$$
\frac{d B}{d T}=\frac{2 k_{B} v^{2}}{c^{2}} \frac{z^{2} e^{z}}{\left(e^{z}-1\right)^{2}}, \text { where } z=\frac{h v}{k T} .
$$

Using the values of the physical constants and the BICEP band centers, the conversion factors are

$$
\frac{d B}{d T}=0.22,0.40,0.48 \frac{\mathrm{MJy} \mathrm{sr}^{-1}}{\mathrm{mK}},
$$

for 100 (95.5), 150 (149.8), and 220 (208.2) GHz bands, respectively, where the true band centers are shown in parentheses. Similarly, the conversion values are $0.016,0.033,0.049,0.10$, 0.22 for WMAP's 23 (22.8), 33 (33.0), 41 (40.7), 61 (60.8), and 94 (93.5) GHz channels, respectively, and are used in Table 2. Figure 13 and the subsequent spectral analysis in this section use BICEP, WMAP, and FDS data in these differential intensity units.



Figure 13. Mean intensity as a function of frequency for the $220 \mathrm{GHz}$ analysis region for WMAP's ten DAs (gray triangles) and BICEP's two boresight maps for each of the three bands (black squares). BICEP's $100 \mathrm{GHz}$ points are shifted to the right $5 \mathrm{GHz}$ from the calculated band centers for clarity. WMAP points come from raw, unfiltered maps, downsampled to $1^{\circ}$ resolution. The noiseless FDS model 8 (Finkbeiner et al. 1999) predicted dust maps (green diamonds) have been beam smoothed, spectral bandpass filtered, and downsampled to $1^{\circ}$ to match BICEP's bands but not polynomial filtered. The fit to Equation (18) using only WMAP points (gray) gives a dust spectral index $\zeta_{d}=5.06$, larger than when BICEP data (blue) are included, which gives $\zeta_{d}=3.70$. The pink and red dashed lines are the individual positive and negative spectral index components from fitting to both WMAP and BICEP points.

(A color version of this figure is available in the online journal.)

Figure 13 shows a plot of the mean and standard error of the mean intensity of the analysis area pixels from both BICEP and WMAP, calculated from each of the ten WMAP DAs and two boresight maps for BICEP's three bands in differential intensity units. BICEP maps have been filtered by the mapmaking process, systematically lowering the intensity values. To correct for this, each BICEP point and error bar has been increased by $11 \%$ (Section 3.3.3). The error bars for the FDS points and other bands come from sky variance, not measurement errors. BICEP has two data points per band that are computed from the two boresight maps. The fact that two 
data points are nearly identical indicates that the systematic uncertainties are not the dominant source of uncertainty for the average intensity of the maps, even for the $220 \mathrm{GHz}$ channels, consistent with the discussion in Section 3.3. WMAP points are from each DA separately, which can indicate the approximate level of residual systematic uncertainty in a given band.

The points in Figure 13 are fit to

$$
I(v)=A_{s} v^{\zeta_{s}}+A_{d} v^{\zeta_{d}}
$$

a simple two-component power-law model where $A_{s, d}$ are the magnitudes of the synchrotron and dust components, and $\zeta_{s, d}$ are the power-law exponents for the synchrotron and dust components. BICEP's points and error bars have been increased by $11 \%$ using the systematic filter correction found in Section 3.3.3. The best-fit spectral index parameters and errors from using only the WMAP points in Equation (18) are $\left[\zeta_{s}, \zeta_{d}\right]=[-0.36,5.06] \pm[0.02,0.33]$, while if BICEP data are included then $\left[\zeta_{s}, \zeta_{d}\right]=[-0.39,3.70] \pm[0.07,0.11]$. For reference, FDS model 8 predicts $\zeta_{d}=3.5$ in the frequency range $23-220 \mathrm{GHz}$ in the same analysis region. From signal simulations, the uncertainty in systematic filter correction could be as large as 5\%; however, this factor has a relatively minor effect on the fit. For example, a 5\% uncertainty in this correction factor leads to an uncertainty in $\zeta_{d}$ of $\sigma_{\zeta_{d}}=1 \%$. This reduced sensitivity to input uncertainty is not only partially due to the nonlinear nature of the fitting model but also due to the WMAP points not suffering from BICEP's filtering bias. Repeating the fit procedure with only one set of boresight maps causes a change in $\zeta_{d}$ of $\sigma_{\zeta_{d}}=2 \%$. Splitting the map into the Gal-bright and Galweak regions gave $\zeta_{d}=[3.81,3.27]$ with $\sigma_{\zeta_{d}}=[0.14,0.15]$, indicating a difference in dust spectral index between the two regions at 2.7 sigma significance.

Therefore, in this case, the uncertainty in this fit itself is larger than the uncertainty due to systematic contamination from weather or telescope systematics, which is larger than the uncertainty due to systematic filtering bias uncertainty. Analyzing the WMAP DAs separately is a way to include systematics from that experiment. However, the separation between points in any WMAP band (see Figure 13) is smaller than the uncertainty in the average value from that band, making systematics minimally important.

The simple two-component model fits the data well; however, additional components are not excluded. The plot shows the importance of measurements at higher frequency bands to fully understand the composition of the ISM. At $96 \mathrm{GHz}$, using the two-component fit parameters, dust makes up $51 \%$ of the total emission, while at $150 \mathrm{GHz}$ it makes up $87 \%$ and at $208 \mathrm{GHz}$ it makes up 97\%. Finkbeiner (2003) gives a template for the full-sky free-free emission based on $H_{\alpha}$ measurements. Taking their input map at 0.0625 resolution, downsampled to $1^{\circ}$ resolution, converted to $\mathrm{MJy} \mathrm{sr}^{-1}$ using the factors given in their Table 1 at $100 \mathrm{GHz}$, the average map pixel value in the $220 \mathrm{GHz}$ analysis region is $0.004 \mathrm{MJy} \mathrm{sr}^{-1}$. Therefore, free-free is approximately 40 times smaller than either the dust or synchrotron component in BICEP maps. Further measurements at intermediate frequencies with finer spacing would also provide useful information about the transition from the synchrotron to dust dominated regime and give more information about other possible emission sources.

\subsection{Polarization Fraction vs. Intensity}

The Galactic magnetic field cannot be measured directly, therefore measurements of the field's effect on the interven-
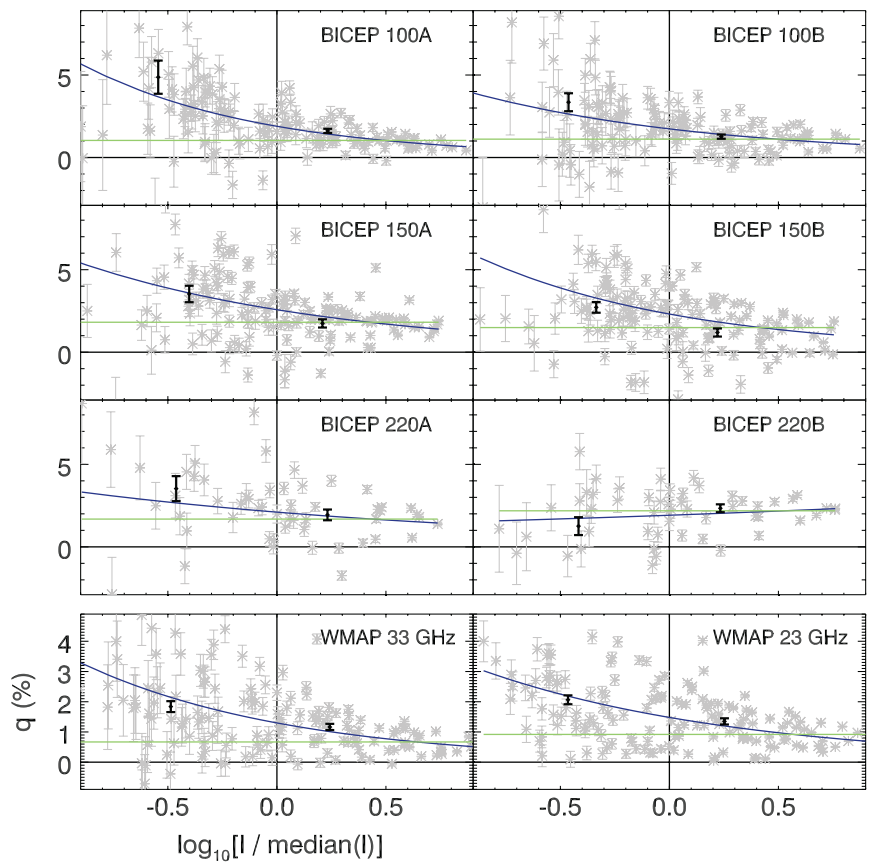

Figure 14. Polarization fraction, $q$, as a function of total intensity, $I$, using 147 one degree Healpix pixels (gray) from BICEP's three bands and two boresight maps along with WMAP 23 and $33 \mathrm{GHz}$ bands. Out of the 147 pixels, 53 were used for the BICEP $220 \mathrm{GHz}$ detectors because of the reduced observed region available at that band. The error bars are those due to the white noise in the maps and do not account for systematic errors due to the atmosphere, instrumental systematics, or filtering. The best-fit line (blue) and constant model (green) are shown. The weighted average and uncertainty (black) of $q$ for pixels less than the median value and greater than the median value clearly show the trend of decreasing polarization fraction as intensity increases.

(A color version of this figure is available in the online journal.)

ing interstellar matter are crucial. The Galactic magnetic field produces polarization on large and small scales across the electromagnetic spectrum. Millimeter-wave polarization measurements probe emission that spans the full extent of the Galactic plane, making it possible to exclude or motivate models for the Galactic magnetic field structure.

\subsubsection{Polarization Fraction Data}

A testable prediction of Galactic magnetic field models is the trend of observed polarization fraction as a function of unpolarized intensity (Miville-Deschênes et al. 2008). To determine if there is a trend and what the nature of the trend may be, Figure 14 shows scatter plots of $q$ versus $I$ for the various BICEP maps with two WMAP channels also shown for comparison. The plotted map pixels for both BICEP and WMAP are from the $100 / 150 \mathrm{GHz}$ analysis area, except for the $220 \mathrm{GHz}$ map pixels, which use the $220 \mathrm{GHz}$ analysis area. The error bars are calculated using the noise per band or DA and the integration time per pixel. Overplotted on the scatter plots is the weighted linear least-squares fit following Equation (14) and the weighted mean $q$. Since the white-noise floor value is used, all of the fits had reduced $-\chi^{2}$ values much greater than one, indicating, to some extent, the total absolute uncertainty, including genuine variation about this trend across the sky. To better approximate the total absolute uncertainty in the resulting fit parameters, the uncertainty for each fit parameter is multiplied by $\sqrt{\chi_{\text {reduced }}^{2}}$, which are the error bars used in Figure 15. It is important to note that all of the BICEP maps detect a trend of decreasing 



Figure 15. $q_{\left(I_{\text {median }}\right)}$ and $\eta$ as a function of frequency using BICEP (red) and WMAP (green) data. All frequencies use the $100 / 150 \mathrm{GHz}$ analysis region except for $220 \mathrm{GHz}$, which uses pixels from the smaller $220 \mathrm{GHz}$ analysis region. The error bars are the quadrature sum of fitting errors and the uncertainty from atmospheric conditions. BICEP's points have been corrected for filtering effects, increased by $37 \%$ and $39 \%$ for $q$ and $\eta$, respectively, based on signal simulations. The trend of increasing $q$ with increasing frequency is apparent, while there is no statistically definitive trend for $\eta$.

(A color version of this figure is available in the online journal.)

polarization fraction with increasing intensity at more than three sigma, $\eta / \Delta \eta>3$, except BICEP $220 \mathrm{GHz}$, which has 10 times fewer feeds than BICEP's 100 or 150 channels.

Figure 14 may not sufficiently support the hypothesis that there is a significant trend of decreasing polarization fraction with increasing intensity as opposed to a constant model for $q$. The difficulty is that the error in polarization fraction increases as the intensity value decreases because the uncertainty in $q$ is proportional to $I^{-1}$, weighting the $q$ values measured more strongly at higher I. However, the fact that there is a slope detected for all bands from $23 \mathrm{GHz}$ to $150 \mathrm{GHz}$ favors a sloped model over a flat one (see Table 2).

\subsubsection{Model Fit Parameters}

Figure 15 shows a plot of the fit parameters from Equation (14) for BICEP's two boresight maps (so there are two data points per band) and WMAP's ten DAs separately (which can give some idea of possible systematic uncertainty in a WMAP band). Table 2 lists the average parameters with uncertainties from three sources and band properties. One type of uncertainty is from the nonlinear regression covariance matrix, and these uncertainties have been increased by $\sqrt{\chi_{\text {reduced }}^{2}}$ to better approximate the true parameter uncertainty and include other noise sources such as genuine spatial variation across the sky.

Second, there is uncertainty due to the dispersion of different maps within a band, the two boresight maps for BICEP, and the various DAs for WMAP. This uncertainty, especially for BICEP, indicates the approximate level of systematic contamination from the atmosphere, instrument thermal properties, and telescope systematics other than spectral gain mismatch. This type of uncertainty is prevalent at all three bands and hinders further quantitative analysis of $\eta$.
Third, there is uncertainty from spectral gain mismatch that is quantified from simulations in Section 2.3 using different atmospheric conditions. This type of uncertainty compared to the other two is only important at $220 \mathrm{GHz}$. Overall, there is a strong detection of $q_{\left(I_{\text {median }}\right)}>0$ at all three bands and a significant detection of $\eta$ at 100 and $150 \mathrm{GHz}$. There is no detection of $\eta$ at $220 \mathrm{GHz}$ due to the combination of all three types of uncertainties exacerbated by the fact that BICEP only has two $220 \mathrm{GHz}$ feeds for only two of the three observing seasons (see Appendix A for more information). Fitting for $\eta$ is somewhat biased by the uncertainty in each pixel being proportional to $I^{-1}$, as the higher intensity pixels tend to dominate the fit. For example, it is difficult to tell whether a third parameter is needed to fit the data properly or whether there are separate effects happening for lower intensity pixels.

The top plot of Figure 15 shows a trend of increasing $q_{\left(I_{\text {median }}\right)}$ versus frequency with a sharp increase between 41 and $150 \mathrm{GHz}$. The two boresight map points per band are close to each other relative to the error bars, indicating that the general level of systematic uncertainties is not the dominant uncertainty for $q_{\left(I_{\text {median }}\right)}$. At higher frequencies where dust emission dominates, the polarization fraction is above $2.5 \%$, rather than below $1.5 \%$ at a lower frequency where synchrotron emission dominates. The polarization fraction is nearly constant as a function of frequency in the dust-dominated bands as predicted by Hildebrand et al. (1999) and expanded upon by Hildebrand \& Kirby (2004), Vaillancourt (2007), and Draine \& Fraisse (2009).

The observations of low polarization fraction for synchrotron or higher relative polarization fraction for dust may seem to be in contradiction to standard theory or observation (Rybicki \& Lightman 1979; Kogut et al. 2007); however, standard values are typical of high Galactic latitude regions, not necessarily in the Galactic plane. In addition, while synchrotron emission theory predicts a maximum polarization fraction near $75 \%$, this has not been observed and values closer to $20 \%$ are commonly found off the plane where the emission efficiency should be the highest. Line-of-sight and beam size effects can further decrease the observed polarization fraction. Unpolarized emission mechanisms, such as free-free or spinning dust emission, contribute to the measured intensity but not to the polarization, lowering the measured polarization fraction. However, Section 3.5 indicates that free-free does not significantly contribute to the BICEP bands and sky coverage region. A comparison to Figure 5 of Kogut et al. (2007), which shows a histogram of polarization fraction from pixels within the Galactic plane, shows that the polarization fraction levels for the WMAP map pixels chosen here are in agreement with the lowest histogram bin of values within the Galactic plane. This is consistent with the BICEP observing region being in the Galactic plane.

The bottom plot of Figure 15 shows $\eta$ as a function of frequency; however, no model of this function is proposed in this paper. The two boresight map points per band show a comparable level of systematic error relative to the statistical error from the error bars. This is consistent with the discussion in Section 3.3 but is approaching an unacceptable level. One important point is that $\eta$ changes the relative positions of the points in $q$ versus frequency. For example, since the average $\eta$ value at $220 \mathrm{GHz}$ is close to zero, the value of $q$ at $220 \mathrm{GHz}$ is relatively constant as a function of total intensity. Therefore, if $q$ were evaluated at a very large value of total intensity and the plot of $q$ versus frequency remade, then it would appear that the polarization fraction increases relative to $150 \mathrm{GHz}$, even though 


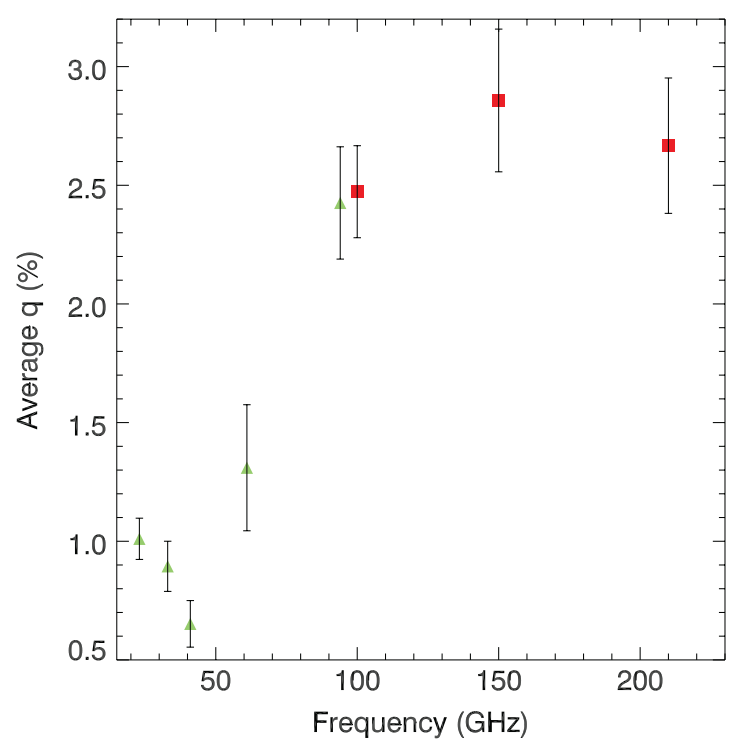

Figure 16. Average and error of $q$ from the $220 \mathrm{GHz}$ analysis region pixels combining all the data at each BICEP (red) and WMAP band (green). This shows a different method of computing $q$ vs. frequency as compared to Figure 15, which uses a model to find the value of $q$, splits the data by boresight angle or DA, and uses a different number of pixels at $220 \mathrm{GHz}$. Once again, a general trend of increasing polarization fraction vs. frequency is found with the minimum $q$ occurring near $40 \mathrm{GHz}$. There is a steep increase in polarization fraction between $60 \mathrm{GHz}$ and $100 \mathrm{GHz}$ with the minimum fraction occurring near $40 \mathrm{GHz}$.

(A color version of this figure is available in the online journal.)

the actual polarization fraction value at $220 \mathrm{GHz}$ was nearly constant.

The weighted mean and standard error of $\eta$ across all bands, assuming the BICEP data points are corrected for filtering, is $\eta=-0.47 \pm 0.05$. A simple, flat polarization model, $\eta=0$, is ruled out as is the simple toy model of unpolarized embedded sources in a uniformly polarized background in the Galaxy, from Section 3.2, $\eta=-1$. A more complicated Galactic magnetic field model is needed to explain the measured value. A comparison of $q$ and $\eta$ between the Gal-bright and Gal-weak regions was performed, but there was no detectable difference between the two regions.

An inherent problem in analyzing the $q$ versus frequency plot is that it depends on the intensity value at which $q$ is evaluated. While evaluating $q$ at the median intensity gives a $q$ value at a moderate level of intensity, other methods, such as computing the average of $q$, effectively result in finding $q$ at the weighted mean intensity, as in Figure 16. The average has two advantages: a model including $\eta$ is not needed and no value of $I$ needs to be chosen at which to evaluate $q$. For this plot, the average from all the data is shown, neglecting different boresight angles and DAs. As opposed to Figure 15, this figure uses only map pixels from the $220 \mathrm{GHz}$ region, simplifying the comparison of $220 \mathrm{GHz}$ versus the other bands. While the minimum $q$ occurs near $40 \mathrm{GHz}$ in this plot, depending on what value of intensity $q$ is evaluated, this frequency can change. The polarization fraction approximately quadruples from $41 \mathrm{GHz}$ to $95 \mathrm{GHz}$, showing a relatively large dependence on frequency. No trend in polarization fraction above $100 \mathrm{GHz}$ is visible, although the error bars do not exclude this possibility. In the end, Figure 15 demonstrates the systematics levels are small compared to the general trend of $q$ versus frequency, as is evident from either Figure 15 or Figure 16 (even if the error bars are larger for Figure 16 because fewer map pixels were used in the average).

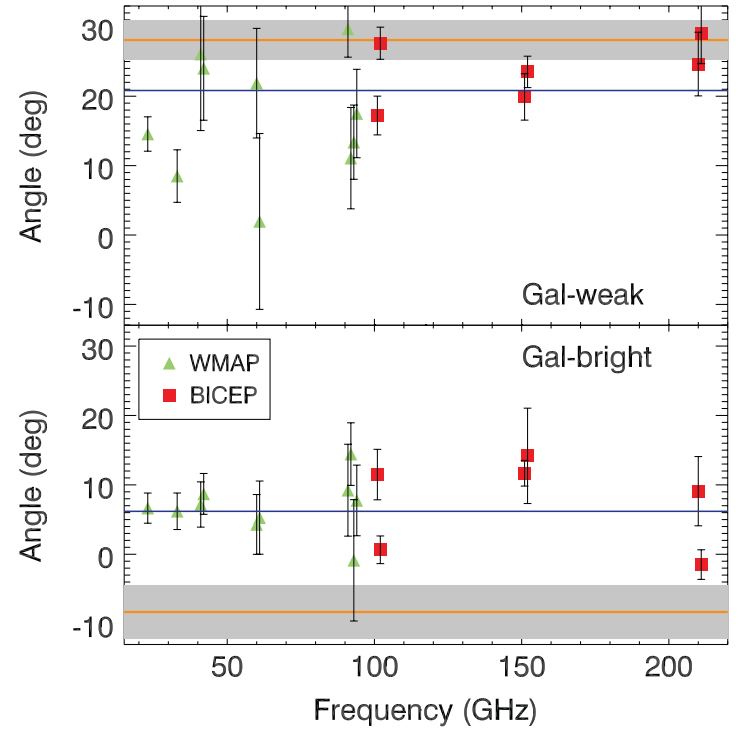

Figure 17. Polarization angle as a function of frequency for BICEP (red squares) and WMAP (green triangles) with the average angle overplotted (blue line) and the average starlight polarization angle (orange line) and uncertainty (gray shaded region) for the Gal-weak region and Gal-bright regions. The polarization angles do not have any trend as a function of frequency in either region showing consistency between the two experiments and different emission mechanisms. There is a net positive rotation of polarization angle from the Gal-bright to Galweak regions for all millimeter-wave bands as well as the starlight polarization angles, indicating a large-scale structure in the Galactic magnetic field.

(A color version of this figure is available in the online journal.)

\subsection{Polarization Angles}

The Galactic magnetic field generally causes polarization angles to be perpendicular to the Galactic plane in the millimeter regime. However, BICEP and WMAP measurements show the magnetic field is not exactly parallel to the plane and changes direction between the two observing regions (Figure 17). The analysis area is split in half by region, Gal-bright (27 pixels) and Gal-weak (26 pixels), and the weighted average polarization angle for each BICEP boresight maps and WMAP DA is found. The two BICEP boresight maps per band show the general level of systematic uncertainty due to excess noise and telescope systematics. WMAP points are from each DA separately, which can be indicative of possible systematic uncertainty in a given band.

The average polarization angle in the Gal-bright region from both BICEP and WMAP is $6.2 \pm 1.7$, whereas the average polarization angle in the Gal-weak region is $20.8 \pm 2.2$ for both BICEP and WMAP. This represents a 5 sigma detection of a difference in polarization angle between the two regions. Note that the BICEP angles have not been corrected for filtering effects.

BICEP and WMAP polarization angles can also be compared to starlight polarization data. Millimeter-wave dust polarization is due to emission from particles that are preferentially aligned perpendicular to the local magnetic field (Lazarian 2007). A complimentary process takes place in the optical band due to absorption, leading to polarization angle that should be rotated $90^{\circ}$ relative to the millimeter-wave polarization. Heiles (2000) compiled a table of stellar polarization measurements and characteristics that can be used to compare polarimetry from the optical and millimeter-wave bands. Out of the 9286 stars, only those with angle errors less than $10^{\circ}$ that are within the BICEP observing region were considered. For a direct 
comparison, the starlight polarization angles have the expected $90^{\circ}$ difference subtracted off. There were 36 stars with starlight polarization measurements in the Gal-bright region giving a weighted average polarization angle of $-8.3 \pm 3.8$, while there were 24 stars in the Gal-weak region giving a weighted average polarization angle of $28^{\circ} .1 \pm 2.8$. One caveat is that the starlight polarization measurements do not exactly track BICEP's and WMAP's measurements in space, as there are multiple stars in some map pixels and none in others. In general, there is better agreement between optical and millimeter-wave polarization angles in the Gal-weak region as opposed to the Gal-bright region.

The polarization direction seen in all millimeter-wave bands and by the starlight polarization measurements increases systematically from the Gal-bright to Gal-weak regions. Since the Gal-bright and Gal-weak regions are separated by $60^{\circ}$ in Galactic longitude, this indicates the Galactic magnetic field has structure on very large scales. Further small-scale analysis is possible, but results are inconclusive due to the uncertainty in BICEP's current polarization angle measurements. As opposed to $q$, there is no difference in the average polarization angle between pixels whose intensity is less than the median intensity and greater than the median intensity. No trend in polarization angle as a function of frequency is detected, showing consistent, independent polarization angle measurements from lower frequencies where synchrotron dominates to high frequency where dust emission dominates.

\subsection{Visual Optical Depth}

In order to compare underlying astronomical objects from the optical to millimeter-wave band, the intensity of the emission can be converted to a common unit used in studies of the interstellar medium, visual optical depth, $A_{V}$. Following Equation (18), the conversion can be calculated as

$$
\frac{d A_{V}}{d T}=r_{\text {dust }} \times \frac{d A_{V}}{d \tau} \times \frac{d \tau}{d I} \times\left.\frac{d B}{d T}\right|_{2.725 \mathrm{~K}},
$$

where the relationship between the emitted intensity and optical depth, assuming an optically thin medium, is given by

$$
I=B\left(1-\exp ^{-\tau}\right) \approx B \tau .
$$

A relationship to millimeter-wave optical depth, $\tau(\lambda)$, derived by Hildebrand (1983) and Dickman (1978) is

$$
A_{V} \approx 1900 \tau(\lambda)\left(\frac{\lambda}{250 \mu \mathrm{m}}\right)^{2} .
$$

Since this calculation is only valid for dust emission and BICEP measures, the total emission from all components, a conversion factor, $r_{\text {dust }}$, must be applied. For example, Section 3.5 showed that at $96 \mathrm{GHz}, 51 \%$ of the emission comes from other sources such as synchrotron radiation. BICEP intensity maps can be converted to $A_{V}$ for the dust component in the map as

$$
\begin{aligned}
\frac{d A_{V}}{d T} \approx & r_{\text {dust }} \times 1900\left(\frac{\lambda}{250 \mu \mathrm{m}}\right)^{2} \times \frac{1}{B_{(20 \mathrm{~K})}} \\
& \times \frac{2 k_{B} v^{2}}{c^{2}} \frac{z^{2} e^{z}}{\left(e^{z}-1\right)^{2}} .
\end{aligned}
$$

Using dust conversion factors of $r_{\text {dust }}=[0.51,0.87,0.97]$ from Section 3.5, assuming a dust temperature of $20 \mathrm{~K}$, substituting in the values of the constants, wavelengths, and BICEP band centers, the conversion factors are

$$
\frac{d A_{V}}{d T} \approx 7.14,3.75,1.44 \frac{\mathrm{A}_{\mathrm{V}}}{\mathrm{mK}},
$$

for 100,150 , and $220 \mathrm{GHz}$, respectively.

BICEP detections span a thermodynamic temperature brightness range from $[0.1,0.3,0.7]$ to $[6,8,25] \mathrm{mK}$ for 100,150 , and $220 \mathrm{GHz}$, respectively. This corresponds to integrated visual optical depths $A_{V}$, using Equation (22), from $[0.7,1.1,1.0]$ to [42, $30,36]$. On average, BICEP probes a component of the ISM which is more diffuse than star-forming regions $\left(A_{V} \gtrsim 10\right)$, but is more dense than the medium sampled by optical polarimetry $\left(A_{V} \lesssim 2\right)$. Due to the relative beam sizes and dust spectrum, BICEP measurements probe approximately the same density medium at local intensity maxima at all three bands. In principle, experiments such as QUaD and Archeops have the potential to probe even denser cloud complexes because of their smaller beam sizes; however, Archeops had higher noise levels than BICEP and both Archeops and QUaD have so far presented their polarization data with angular resolution $\approx 0.5$, similar to BICEP.

\section{DISCUSSION}

Continuum polarimetry results can be interpreted with the aid of a model in which the magnetic field for a given patch of sky is nearly constant in magnitude but has an angular structure which is a superposition of a uniform and a random component (Jones 1989; Miville-Deschênes et al. 2007). The very simplest models, such as a completely uniform magnetic field or a completely random one, are ruled out by BICEP and WMAP, which show a statistically significant trend of decreasing $Q$ polarization fraction versus increasing intensity (Section 3.6).

The detection of dust polarization with Archeops, WMAP, $\mathrm{QUaD}$, and BICEP constrains the degree of order in the Galactic magnetic field on large scales. An ordered magnetic field nearly parallel to the plane is detected at all millimeterwave frequencies. The mean polarization angles are nearly constant as a function of frequency, but systematically change direction between BICEP's two Galactic regions. This change in direction is also found in starlight polarization measurements and confirms structure in the Galactic magnetic field on scales greater than $60^{\circ}$ in Galactic longitude.

The average degree of polarization observed in the integrated emission from star-forming cores $\left(A_{V} \gtrsim 30\right) p \lesssim 1.5 \%$ (Stephens et al. 2011) is similar to the degree of polarization observed with BICEP (Section 3.6). However, this coincidence does not imply that the observed BICEP polarization arises from a superposition of unresolved star-forming cores, with no significant polarized component emitted by the diffuse medium. While there is some evidence for coherence in the magnetic field across star-forming molecular cloud complexes up to $100 \mathrm{pc}$ in size ( $\mathrm{Li}$ et al. 2009), as a whole, those complexes and cores have a nearly random distribution of magnetic field directions and there is no evidence for coherence in the dense medium on larger scales (Glenn et al. 1999; Stephens et al. 2011). The contribution from these star-forming complexes to BICEP maps would consist of polarization with a high angular disorder, averaging to very low polarization when the beam encompasses multiple complexes. For example, a BICEP $150 \mathrm{GHz} 0.6$ beam corresponds to $50 \mathrm{pc}$ for a typical Galactic source at a distance of $5 \mathrm{kpc}$ in the Gal-bright field, and hence poorly 
resolves molecular cloud complexes. Consequently, because BICEP observes a substantial degree of polarization over the whole Galactic plane at $\sim 1^{\circ}$ resolution on average, BICEP must sample a medium outside star-forming cores, one with an embedded magnetic field that retains a significant component ordered on the Galactic scale.

On the other hand, BICEP rules out a model where no additional polarization intensity comes from the higher density ISM. The polarization fraction drops somewhat as surface brightness increases, $q \propto I^{-0.47}$, but the decline is more shallow than $q \propto I^{-1}$ as predicted by the toy model of Section 3.2 having bright unpolarized sources embedded in a polarized medium. Therefore, BICEP measurements probe aligned grains and magnetic fields over the full range of observed column densities approaching star-forming cores. Jones (1989) and Fosalba et al. (2002) find an equivalent power-law exponent from starlight data closer to $\eta_{\mathrm{em}}=-0.25$, implying a more ordered magnetic field than what is implied from millimeter-wave measurements. A caveat to this comparison is those previous works analyzed total polarization fraction $p$, which does not necessarily have the same functional relationship as $q$. No attempt to directly model $q$ using magnetic fields is included in this paper.

The detection of statistically significant polarization across the Galactic plane at all intensity levels shows that there are aligned dust grains across the entire map. The $\eta$ parameter indicates the nature of the Galactic magnetic field and the disorder within the beam and along the line of sight. The polarization fraction $q$ increases as a function of frequency, which was somewhat unexpected; other work has indicated that the opposite can be true at higher latitudes due to the much larger polarization fraction intrinsic to the synchrotron emission compared to dust polarization (Kogut et al. 2007). In contrast, BICEP has shown that dust emission can have a higher polarization fraction in the Galactic plane. This could indicate unpolarized emission mechanisms such as spinning dust play a more important role in the map regions analyzed here or it could be an indication that material-emitting synchrotron radiation exists in more randomized Galactic magnetic field regions on average, as opposed to dust grains which may lie in more ordered field regions.

The observations discussed here are consistent with a Galactic magnetic field whose structure and order vary inversely with density. The increasing disorder in denser components of the ISM may be due to gravitational or dynamical accumulation of gas and/or feedback from star formation. The high angular resolution and sensitivity of the Planck (Tauber et al. 2010) $350 \mathrm{GHz}$ polarization channels should be able to map this structure with greater precision to discriminate among more detailed models for the neutral ISM.

\section{CONCLUSION}

BICEP's Galactic observations have added new information and insight into Galactic physics, while also confirming previous measurements. BICEP samples an intermediate optical depth of the ISM and polarization is detected everywhere within two degrees of the Galactic plane with values ranging up to a few percent at 100, 150, and $220 \mathrm{GHz}$. BICEP detects a significant trend of decreasing polarization fraction as intensity increases in the maps and increasing polarization fraction as a function of frequency. Polarization angles were found to be consistent from $23 \mathrm{GHz}$ to $220 \mathrm{GHz}$ and in general agreement with polarization angles measured by stars in our observing regions. Adding $220 \mathrm{GHz}$ capability to BICEP helped to refine the understanding of in-plane foreground emission. BICEP data have shown astronomical foregrounds to be complex, and simply modeling polarization as a percentage of unpolarized intensity is insufficient. While polarized foreground models in the Galactic plane are becoming more precise, less is known at higher latitudes and it is not obvious that one can extrapolate models from the plane to these latitudes. Future CMB polarimeters are poised to build upon BICEP's results.

We especially recognize Andrew Lange whose passing has deeply affected everyone on the team and who never saw the culmination of this project. Without his guidance and friendship this paper would not have been possible.

BICEP is supported by NSF Grant OPP-0230438, Caltech Discovery Fund, Caltech President's Fund PF-471, JPL Research and Technology Fund, and the late J. Robinson. We thank our colleagues in the ACBAR, Boomerang, QUaD, Bolocam, POLARBEAR, and SPT collaborations for advice and helpful discussions, Kathy Deniston for logistical and administrative support, and the South Pole Station staff for their support. We gratefully acknowledge support by the NASA Graduate Fellowship program (E.M.B. and H.C.C.), the John B. and Nelly Kilroy Foundation (J.M.K.), the NSF PECASE Award AST0548262 (B.G.K.), the US DOE contract to SLAC No. DEAC02-76SF00515 (C.L.K. and J.E.T.), KICP (C.P. and C.S.), and the NASA Science Mission Directorate via the US Planck Project (G.R.). We also thank the thorough and comprehensive work of the referee that has tremendously improved the quality of the final version of this paper.

\section{APPENDIX A}

\section{BICEP $220 \mathrm{GHz}$ CHANNELS}

Prior to the second observing season, two feeds designed to observe through the $220 \mathrm{GHz}$ atmospheric transmission window were installed on BICEP. These feeds represent the first attempt to observe $\mathrm{CMB}$ and Galactic polarization in this frequency band using PSBs. Due to the relative sensitivity of a $220 \mathrm{GHz}$ channel to thermal dust emission and its polarization over lower frequency channels, the $220 \mathrm{GHz}$ feeds serve a threefold purpose. First, to constrain the polarization and intensity of the three main millimeter-wave sources (synchrotron, thermal dust, and the CMB) solely from BICEP observations, without an ad hoc foreground model, requires three frequency bands. Second, thermal dust emission and its polarization increase understanding of the physics of the Galaxy. Finally, it was unclear whether the CMB B-modes, BICEP's primary science target, would be contaminated by foreground emission, and would need to be modeled and removed. The $220 \mathrm{GHz}$ feeds can carry more weight, per feed, than the other two bands for measurement of dust polarization due to the steep spectral index of millimeter-wave dust emission. More $220 \mathrm{GHz}$ feeds were planned to be added in 2008 but logistical constraints at the South Pole in 2008 ultimately prevented this, limiting the experiment to using two feeds for the two final observing seasons.

Emission from the Galaxy has the potential to contaminate the faint CMB B-mode signal in all observing fields. Since dust emission follows a thermal spectrum, by monitoring polarized interstellar dust at higher frequency than the primary B-mode bands (100 and $150 \mathrm{GHz}$ ), it is possible to monitor dust contamination with higher signal-to-noise per feed. While BICEP's 


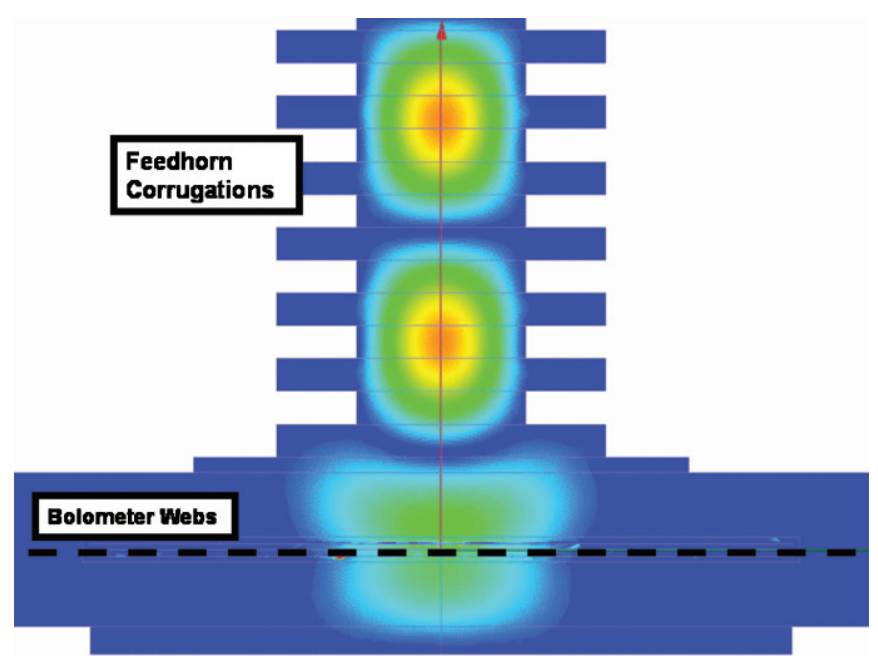

Figure 18. HFSS simulation of the $220 \mathrm{GHz}$ coupling feedhorn to $150 \mathrm{GHz}$ bolometer. The color scale represents the electric field intensity. The phase was chosen to maximize the field intensity at the bolometer webs. The shape of the coupling feedhorn and distance between the last corrugation in the feedhorn to the position of the bolometer webs is optimized to increase the coupling efficiency and minimize the cross-polarization response.

(A color version of this figure is available in the online journal.)

CMB region, located at high Galactic latitude, certainly exhibits minimal dust column density and therefore low-intensity millimeter-wave emission, this does not imply it will exhibit low-polarized emission due to the details of magnetic grain alignment (Lazarian 2007). Little is known about the polarization properties of thermal dust emission in the high-frequency bands above $100 \mathrm{GHz}$ used for CMB observations. BICEP's $220 \mathrm{GHz}$ feeds are a unique link between the low-frequency CMB polarimetry of WMAP and the high-frequency survey of the Archeops experiment, from centimeter wavelengths to the submillimeter. The combination of WMAP, QUaD, BICEP, and Archeops polarization observations of the Galactic plane covers more than a decade of frequency.

BICEP PSBs were only fabricated for 100 and $150 \mathrm{GHz}$ operations. The $220 \mathrm{GHz}$ pixels therefore were modified from $150 \mathrm{GHz}$ PSBs following the first season of observations. The $220 \mathrm{GHz}$ feed installation consisted of replacing two $150 \mathrm{GHz}$ feedhorn stacks with a set of $220 \mathrm{GHz}$ feedhorn stacks. Each feedhorn stack consists of three separate feedhorn pieces, all of which used the same outer-forms as the $150 \mathrm{GHz}$ feedhorns. Two of the feedhorn inner-forms were made with a profile similar to the $150 \mathrm{GHz}$ feedhorns. The most difficult part of the design process was to optimize the feedhorn that couples the radiation to the bolometer. In this case, the $220 \mathrm{GHz}$ coupling feedhorn and corrugation profile had to be matched to the existing $150 \mathrm{GHz}$ bolometer and housing. Using HFSS, ${ }^{17}$ the feedhorn-to-bolometer housing coupling, co-polar beam shape, total throughput, and cross-polarization beam response were optimized. Specifically, a straight $220 \mathrm{GHz}$ feedhorn profile inserted into the $150 \mathrm{GHz}$ housing resulted in the best overall simulated performance (Figure 18). The lower frequency cutoff of the $220 \mathrm{GHz}$ band is defined by the feedhorn waveguide cutoff while the higher cutoff is defined by a set of metal mesh filters, borrowed from the ACBAR experiment (Runyan et al. 2003).

\footnotetext{
17 Ansoft's HFSS: http://www.ansoft.com/products/hf/hfss/
}

\section{APPENDIX B TELESCOPE CHARACTERIZATION}

Summarized here are the characterizations of parameters in Table 1 not mentioned previously. For more information, see Takahashi et al. (2010). The detector polarization angle, $\psi$, is measured with a 0.7 uncertainty across all three bands, while the relative angle uncertainty in the pair is measured with a 0.1 accuracy (Takahashi et al. 2010). Nominally, this is the mechanical orientation of the bolometer web in the focal plane; however, in practice, this is a parameter characterized after deployment for more precise analysis. The ability to measure this parameter with such low statistical and systematic error is one of BICEP's design advantages.

The average cross-polarization response, $\epsilon$, measures the relative magnitude of the residual signal in the orthogonal or cross-polarization direction given a purely polarized input in the co-polar direction. The HFSS simulations predicted the $220 \mathrm{GHz}$ feeds should have an $\epsilon$ less than 0.07, similar to the measured value. Depolarization is larger for the $220 \mathrm{GHz}$ channel due to the coupling effects between the $220 \mathrm{GHz}$ coupling feedhorn and the bolometer and housing optimized for $150 \mathrm{GHz}$ observations. The resulting polarization efficiency, $\gamma$, is the loss of polarization signal compared to the unpolarized signal used for calibration. $\gamma$ directly affects the observed polarization fraction; however, it has been measured to $2 \%$ uncertainty, which is much smaller than other errors in the measurement.

The optical efficiencies (OEs) were derived by taking load curves while observing blackbody radiation at different temperatures and give the total end-to-end sensitivity on the sky. While this parameter is not used in the mapmaking pipeline, it is implicitly included in the telescope noise estimates. The OEs for the last observing season were $20.8 \%, 19.8 \%$, and $15.8 \%$ for 100,150 , and $220 \mathrm{GHz}$, respectively. HFSS simulations for the $220 \mathrm{GHz}$ feeds predicted a coupling efficiency over $98 \%$ (similar to the 100 and $150 \mathrm{GHz}$ feeds) with $2 \%$ reflected power. The $\mathrm{OE}$ of the feeds that were converted from $150 \mathrm{GHz}$ to $220 \mathrm{GHz}$ declined by an average of $15 \%$, while the other feeds did not change between seasons. The simulated transmission and reflectivity did not include the lenses, which were optimized for $125 \mathrm{GHz}$ operation (midway between 100 and $150 \mathrm{GHz}$ ), not $220 \mathrm{GHz}$.

The beam functions, $P(\Omega)$, were measured using a circularly polarized broadband noise source for 100 and $150 \mathrm{GHz}$ feeds and Jupiter for $220 \mathrm{GHz}$ feeds. The beam response functions are fit with an elliptical Gaussian giving an average beam FWHM of $0.93,0.60$, and 0.42 for 100,150 , and $220 \mathrm{GHz}$, respectively. The differential beam size and ellipticities are very small for BICEP and do not affect the results of this paper. Both polarized and unpolarized sidelobe responses are characterized by observations of a broadband source and are determined to be negligible for this analysis.

The absolute telescope pointing is calculated from observations of multiple stars taken by an optical camera mounted to the telescope. The absolute pointing is the same as used in Chiang et al. (2010) and is independent of a given feeds transmission band. Relative radio pointing for each feed is calibrated using individual maps of CMB temperature anisotropy. The differential pointing mismatch between PSB pairs has similar values across all three bands, with a mean of $0.0041,0.0046$, and $0.0047(1.0 \%, 1.8 \%, 2.6 \%$ of the beam size) for 100,150 , and $220 \mathrm{GHz}$, respectively. The $220 \mathrm{GHz}$ intensity maps show some 
small evidence for differential pointing between the two PSBs within a feed and between the two feeds.

Reflections in the optical system created a "ghost image" opposite to the primary image with respect to the boresight for each feed. The average ghost image power, as determined by observations of the Moon, was $0.41 \%, 0.50 \%$, and $1.3 \%$ for 100,150 , and $220 \mathrm{GHz}$, respectively, relative to the primary image. The magnitudes of the ghost beam images relative to the primary image are nearly identical for a given pair of PSBs in a feed, giving power differences of $0.02 \%, 0.04 \%, 0.04 \%$ for the 100, 150, and $220 \mathrm{GHz}$ bands, respectively. Since the Moon's high intensity lowers the responsivity of the detectors, the ghost image systematics given here are upper limits. These differences are below the noise level in the Galactic polarization maps for all three bands. While this effect has been measured well using a bright source such as the Moon, there is no evidence for ghost effects in the Galactic maps.

A miscalibration of the time-domain impulse response, $K(t)$, would act like a beam size mismatch leaking intensity to polarization. However, the time-domain response for each of the three bands is measured to a very high precision and deconvolved from the raw $50-\mathrm{Hz}$-sampled timestream. There is no evidence for time constant mismatch from either the Galaxy data or CMB data.

\section{REFERENCES}

Ade, P. A. R., Pisano, G., Tucker, C., \& Weaver, S. 2006, Proc. SPIE, 6275, $62750 \mathrm{~T}$

Amblard, A., Cooray, A., \& Kaplinghat, M. 2007, Phys. Rev. D, 75, 083508

Bennett, C. L., Halpern, M., Hinshaw, G., et al. 2003, ApJS, 148, 1

Benoît, A., Ade, P., Amblard, A., et al. 2004, A\&A, 424, 571

Bock, J., Cooray, A., Hanany, S., et al. 2008, arXiv:0805.4207

Burn, B. J. 1966, MNRAS, 133, 67

Chiang, H. C., Ade, P. A. R., Barkats, D., et al. 2010, ApJ, 711, 1123

Chuss, D. T., Davidson, J. A., Dotson, J. L., et al. 2003, ApJ, 599, 1116

Culverhouse, T., Ade, P., Bock, J., et al. 2010, ApJ, 722, 1057

Davis, L., Jr., \& Greenstein, J. L. 1951, ApJ, 114, 206

Dickman, R. L. 1978, ApJS, 37, 407

Dodelson, S., Easther, R., Hanany, S., et al. 2009, in Astronomy, Vol. 2010, astro2010: The Astronomy and Astrophysics Decadal Survey, 67

Dotson, J. L., Vaillancourt, J. E., Kirby, L., et al. 2010, ApJS, 186, 406

Draine, B. T., \& Fraisse, A. A. 2009, ApJ, 696, 1

Duband, L., Hui, L., \& Lange, A. 1990, Cryogenics, 30, 263

Dunkley, J., Amblard, A., Baccigalupi, C., et al. 2009, in AIP Conf. Ser. 1141, CMB Polarization Workshop: Theory and Foregrounds, ed. S. Dodelson et al. (Melville, NY: AIP), 222

Eriksen, H. K., Dickinson, C., Lawrence, C. R., et al. 2006, ApJ, 641, 665

Eriksen, H. K., Jewell, J. B., Dickinson, C., et al. 2008, ApJ, 676, 10

Finkbeiner, D. P. 2003, ApJS, 146, 407

Finkbeiner, D. P., Davis, M., \& Schlegel, D. J. 1999, ApJ, 524, 867

Fixsen, D. J., \& Mather, J. C. 2002, ApJ, 581, 817
Fosalba, P., Lazarian, A., Prunet, S., \& Tauber, J. A. 2002, ApJ, 564, 762

Glenn, J., Walker, C. K., \& Young, E. T. 1999, ApJ, 511, 812

Hamaker, J. P., \& Bregman, J. D. 1996, A\&AS, 117, 161

Hansen, F. K., Banday, A. J., Eriksen, H. K., Górski, K. M., \& Lilje, P. B. 2006, ApJ, 648, 784

Heiles, C. 2000, AJ, 119, 923

Hildebrand, R., \& Kirby, L. 2004, in ASP Conf. Ser. 309, Astrophysics of Dust, ed. A. N. Witt, G. C. Clayton, \& B. T. Draine (San Francisco, CA: ASP), 515

Hildebrand, R. H. 1983, QJRAS, 24, 267

Hildebrand, R. H. 1988, Astrophys. Lett. Commun., 26, 263

Hildebrand, R. H., Dotson, J. L., Dowell, C. D., Schleuning, D. A., \& Vaillancourt, J. E. 1999, ApJ, 516, 834

Hiltner, W. A. 1951, ApJ, 114, 241

Jarosik, N., Barnes, C., Greason, M. R., et al. 2007, ApJS, 170, 263

Jones, T. J. 1989, ApJ, 346, 728

Jones, T. J. 2003, New Astron. Rev, 47, 1123

Jones, W. C., Bhatia, R., Bock, J. J., \& Lange, A. E. 2003, Proc. SPIE, 4855, 227

Jones, W. C., Montroy, T. E., Crill, B. P., et al. 2007, A\&A, 470, 771

Kamionkowski, M., Kosowsky, A., \& Stebbins, A. 1997, Phys. Rev. D, 55, 7368

Keating, B. G., Ade, P. A. R., Bock, J. J., et al. 2003a, Proc. SPIE, 2855, 227

Keating, B. G., O'Dell, C. W., Gundersen, J. O., et al. 2003b, ApJS, 144, 1

Kogut, A., Dunkley, J., Bennett, C. L., et al. 2007, ApJ, 665, 355

Larson, D. L., Eriksen, H. K., Wandelt, B. D., et al. 2007, ApJ, 656, 653

Lazarian, A. 2007, J. Quant. Spectrosc. Radiat. Transfer, 106, 225

Leach, S. M., Cardoso, J.-F., Baccigalupi, C., et al. 2008, A\&A, 491, 597

Li, H., Dowell, C. D., Goodman, A., Hildebrand, R., \& Novak, G. 2009, ApJ, 704, 891

Martin, P. G., \& Whittet, D. C. B. 1990, ApJ, 357, 113

Mather, J. C., Cheng, E. S., Cottingham, D. A., et al. 1994, ApJ, 420, 439

Matthews, B. C., McPhee, C. A., Fissel, L. M., \& Curran, R. L. 2009, ApJS, 182,143

Miville-Deschênes, M., Lagache, G., Boulanger, F., \& Puget, J. 2007, A\&A, 469,595

Miville-Deschênes, M., Ysard, N., Lavabre, A., et al. 2008, A\&A, 490, 1093

Novak, G., Chuss, D. T., Renbarger, T., et al. 2003, ApJ, 583, L83

Page, L., Hinshaw, G., Komatsu, E., et al. 2007, ApJS, 170, 335

Ponthieu, N., Macías-Pérez, J. F., Tristram, M., et al. 2005, A\&A, 444, 327

Runyan, M. C., Ade, P. A. R., Bhatia, R. S., et al. 2003, ApJS, 149, 265

Rybicki, G. B., \& Lightman, A. P. 1979, Radiative Processes in Astrophysics (New York: Wiley)

Seljak, U., \& Zaldarriaga, M. 1997, Phys. Rev. Lett., 78, 2054

Stephens, I. W., Looney, L. W., Dowell, C. D., Vaillancourt, J. E., \& Tassis, K. 2011, ApJ, 728, 99

Takahashi, Y. D., Ade, P. A. R., Barkats, D., et al. 2010, ApJ, 711, 1141

Tauber, J. A., Mandolesi, N., Puget, J.-L., et al. 2010, A\&A, 520, A1

Tucci, M., Martínez-González, E., Vielva, P., \& Delabrouille, J. 2005, MNRAS, 360,935

Vaillancourt, J. E. 2007, in Sky Polarisation at Far-Infrared to Radio Wavelengths: The Galactic Screen before the Cosmic Microwave Background, ed. M.-A. Miville-Deschênes \& F. Boulanger (EAS Pub. Ser. Vol. 23; Les Ulis: EDP Sciences), 147

Weiler, K. W. 1973, A\&A, 26, 403

Whittet, D. C. B. 1992, Dust in the Galactic Environment (London: Taylor and Francis)

Wiebe, D. S., \& Watson, W. D. 2004, ApJ, 615, 300

Yoon, K. W., Ade, P. A. R., Barkats, D., et al. 2006, Proc. SPIE, 6275, 62751K 